\title{
Signatures of Genetic Variation in Human microRNAs Point to Processes of Positive Selection and Population-specific Disease Risks
}

\section{Pablo Villegas-Mirón}

IBE: Instituto de Biologia Evolutiva

\section{Alcia Gallego}

Severo Ochoa Molecular Biology Centre: Universidad Autonoma de Madrid Centro de Biologia Molecular

Severo Ochoa

\section{Jaume Bertranpetit}

IBE: Instituto de Biologia Evolutiva

\section{Hafid Laayouni}

IBE: Instituto de Biologia Evolutiva

\section{Yolanda Espinosa Parrilla ( $\square$ yolanda.espinosa@umag.cl)}

University of Magallanes: Universidad de Magallanes https://orcid.org/0000-0002-5441-3113

\section{Research Article}

Keywords: microRNA, human populations, positive selection, cancer, post-transcriptional regulation

Posted Date: June 9th, 2021

DOl: https://doi.org/10.21203/rs.3.rs-578153/v1

License: (c) (1) This work is licensed under a Creative Commons Attribution 4.0 International License. Read Full License

Version of Record: A version of this preprint was published at Human Genetics on March 6th, 2022. See the published version at https://doi.org/10.1007/s00439-021-02423-8. 


\section{Abstract}

The occurrence of natural variation in human microRNAs has been the focus of numerous studies during the last twenty years. Most of them have been centered on the role of specific mutations in disease, while a minor proportion seek to analyse microRNA diversity in the genomes of human populations. We investigate the latest human microRNA annotations in the light of the most updated catalog of genetic variation provided by the 1000 Genomes Project. By means of the in silico analysis of microRNA variants we show that the level of evolutionary constraint of these sequences is governed by the interplay of different factors, like their evolutionary age or genomic location. The role of mutations in the shaping of microRNA-driven regulatory interactions is emphasized with the acknowledgement that, while the whole microRNA sequence is highly conserved, the seed region shows a pattern of higher genetic diversity that appears to be caused by the dramatic frequency shifts of a fraction of human microRNAs. We highlight the participation of these microRNAs in population-specific processes by identifying that not only the seed, but also the loop, are particularly differentiated regions among human populations. The quantitative computational comparison of signatures of population differentiation showed that candidate microRNAs with the largest differences are enriched in variants related to gene expression levels (eQTLs), selective sweeps and pathological processes. We explore the implication of these evolutionary-driven microRNAs and their single nucleotide variants in human diseases, such as different types of cancer, and discuss their role in population-specific disease risk.

\section{Introduction}

MicroRNAs (miRNAs) are short ( 22 nucleotides) single-stranded regulatory non-protein-coding RNAs that perform a post-transcriptional negative control of the expression of more than $60 \%$ of the whole human genome (Friedman et al. 2009). They are involved in the control of almost every cellular process, including development, differentiation, proliferation and apoptosis, and present important roles in diseases. They are transcribed by RNA polymerase II as primary sequences, which are later processed by the Drosha-DGCR8 and Dicer protein complexes into a miRNA duplex formed by two mature miRNA strands, $5 p$ and $3 p$ (Ha et al. 2014). This mature molecule is then loaded onto an AGO protein forming the RNA-induced silencing complex (RISC), promoting the RNA silencing by translational repression or mRNA degradation. Target gene repression is accomplished by the partial sequence complementarity between the target mRNA and the miRNA. In this interaction, a perfect match between the miRNA seed region, expanded across nucleotides 2-8 of the $5^{\prime}$ extreme, and the target site, usually located within the mRNA $3^{\prime}$ untranslated region, is needed (Lewis et al. 2005; Grimson et al. 2007; Bartel et al. 2009; Berezikov 2011). Other positions of the mature sequence also participate in the mRNA binding, like the 3 ' supplementary and compensatory sites, that enhance the seed-matched binding efficiency (Grimson et al. 2007; Friedman et al. 2009; Bartel 2018).

miRNAs have experienced multiple periods of fast turn over and lineage-specific expansions through their evolutionary trajectory (Lu et al. 2008; Iwama et al. 2012). Most of the current human miRNAs originated in two accelerated peaks of miRNA expansion that are reported during mammalian evolution: the first 
peak of new miRNAs was located at the initial phase of the placental radiation, while the second and highest peak was observed at the beginning of the simian lineage, that originated more than a half of the current repertoire (Iwama et al. 2012; Santpere et al. 2016). These miRNA expansions were implicated in the acquisition of new regulatory tools that have been directly linked with animal complexity and evolutionary innovations across all lineages (Hertel et al. 2006; Heimberg et al. 2008; Wheeler et al. 2009).

miRNAs can be found either in intergenic regions or being hosted by other elements, like protein-coding and non-protein coding genes or repetitive elements like transposons. These are the genomic contexts where hairpin-like transcripts initially emerge and are gradually shaped by evolution until they become functional miRNAs (Berezikov 2011). Differences in the genomic environment and location of miRNAs are associated with different evolutionary properties. For example, in França et al. 2016 the authors show the association of the age of the host gene with the breadth expression and evolutionary trajectory of recently emerged hosted miRNAs. Duplication events are one of the main sources of new miRNAs. These can be found close to each other when the duplication is local, forming clusters that are found to be evolutionary related and functionally implicated in similar regulatory pathways (Wang et al. 2016). The origin of miRNAs and their target sites are tightly related to the dynamics of transposable elements (TE). These are sequences that jump, replicate, and insert in other parts of the genome, generating mutations. However, apart from the damaging consequences of these changes, they can also incorporate new functional regions in other genomic environments (like miRNA target sites) and modify regulatory networks (Feschotte 2008; Chuong et al. 2017). According to some authors (Piriyapongsa et al. 2007; Qin et al. 2015; Petri et al. 2019), the expansion of new miRNAs in the primate lineage gave birth to a great number of TE-derived miRNAs, highlighting the importance of transposons as a source of genomic innovation.

The computational analysis of human genetic variation has traditionally been focused on protein-coding genes, being non-protein-coding sequences neglected from this kind of studies. However, in recent years, several reports have paid more attention to the consequences of naturally occurring variation in miRNAs (Cammaerts et al. 2015). A signature of purifying selection shapes the miRNA diversity worldwide, revealing that human miRNAs are highly conserved sequences that rarely accept changes (Quach et al. 2009), indeed miRNA expression and functionality are usually tightly subjected to the presence of variants within (Quach et al. 2009) and outside (Borel et al. 2011) their hairpin. Sequence changes in the premature and loop regions might generate distortions in their folding and affect the expression and maturation of the primary sequences (Fernandez et al. 2017). Moreover, the occurrence of changes in the mature region and the seed, which outstands as the most conserved region of the hairpin, can dramatically affect the recognition of their target genes, which is also affected by the presence of variants in their target sites (Muiños-Gimeno et al. 2009; Gong et al. 2012; Li et al. 2012; Hill et al. 2014; Gallego et al. 2016; He et al. 2018). All these changes might induce massive rewirings of the miRNA regulatory networks and alter the downstream processes, inducing gene expression changes and phenotypic variation that might degenerate in pathogenic processes (Sethupathy and Collins 2008; Rawlings-Goss et al. 2014; Torruella-Loran et al. 2016; Ghanbari et al. 2017; Grigelioniene et al. 2019, Torruella-Loran et al. 2019), but also be the origin of genetic innovations responsible for phenotypic 
adaptations (Lopez-Valenzuela et al. 2012; Lu et al. 2012). Several authors have reported populationspecific variants that affect different dimensions of the miRNA functionality (Saunders et al. 2007; Torruella-Loran et al. 2016) and their target sites (Li et al. 2012) and might be involved in adaptation processes. More recently, it has been reported a clear signal of adaptive evolution in a metabolic-related miRNA responsible for adaptations to past famine periods (Wang et al. 2020).

In this study we revisited the genomic and conservation patterns of the most complete human miRNA catalog to date and performed a comprehensive computational analysis of their diversity patterns worldwide, considering the factors that might contribute the most to the configuration of this variation. We finally studied the population differences and putative positive selection signals of the variable miRNAs, and looked at the potential consequences of this variation in terms of human diseases and recent adaptation.

\section{Results}

\section{The genomic context of miRNAs is associated with their evolutionary age}

To study the recent evolutionary history of human miRNA genes a total of 1918 precursor miRNAs and their mature sequences (miRBase v.22, March 2018) were considered, from which 1904 remained after liftOver conversion to the hg19 assembly (Fig. 1a, Supplementary Table S1 and Fig. S1). First, we classified these 1904 miRNAs in groups of conservation, according to their evolutionary age, by adapting the categories from Iwama et al. (2013) and Santpere et al. (2016). In total, 1623 (85.2\%) miRNAs were classified in four different conservation categories: Primates (985, 51.7\%), Eutherians (421, 22.1\%), Metatheria-Prototheria $(63,3.3 \%)$ and conserved beyond mammals $(154,8 \%)$. The remaining miRNAs $(281,14.8 \%)$ could not be classified due to the absence of data or discrepancies between studies and were excluded from the subsequent analyses (Supplementary Table S1).

Next, we classified miRNAs in different genomic contexts by identifying the different elements that overlap their precursor sequences. According to GENCODE 19 (v.29) we found that 483 ( 25\%) miRNAs fell in intergenic regions (Intg), while 1421 ( 75\%) were located either within protein-coding genes (PC) $(1217,63.9 \%)$ or long non-coding RNAs (IncRNA; LNC) (204, 10.7\%), either presenting a single or multiple overlapping host genes. In our dataset we found that 856 (60\%) intragenic miRNAs (PC and IncRNA) overlapped introns of the host sequence, while 545 (38\%) were located within exonic regions. The remaining $20(\sim 1 \%)$ showed a mixture of intronic/exonic locations (Supplementary Table S1). Further, we used the last release of the RepeatMasker database (Smit et al. 2013-2015) to identify the different forms of transposable elements (TEs) and repetitive sequences that host miRNAs. We found $660(35 \%)$ miRNAs overlapping TEs alone or in combination with other genes, while the remaining 1244 (65\%) were either unmasked or overlapping other forms of repetitive sequences and genes. Interestingly, we found a strong correlation between the frequencies of the TE-hosting miRNAs and their evolutionary age, being the primate-specific group the one with the highest presence of miRNAs in this context (440, 23.1\%; Fig. 1b). Alu (67, 6.8\%), L1 (66, 6.7\%), TcMar (42, 4.2\%) and the LTR elements ERV1 and ERVL (36, 3.6\%) were 
found mainly among the primate-specific miRNAs, while hAT $(14,3.3 \%)$ and $L 2(28,6.6 \%)$ elements were also present in the eutherian group (Supplementary Table S2). It is of interest to note that the contribution of MIR $(101,15.3 \%)$ and DNA elements like TcMar $(48,7.2 \%)$ and hAT $(54,8.1 \%)$ families to the miRNA context is higher than to the whole genome (Supplementary Fig. S2a).

We found that the genomic context increased in complexity when different elements appeared hosting the same miRNA simultaneously. We studied the integrated hosting of miRNAs across the conservation groups considering the different combinations of elements (Fig. 1c, Supplementary Table S3). This shared hosting evidences the two main sources of miRNAs: PC genes $(796 ; 41.8 \%)$ and TEs $(193 ; 10.2 \%)$, with 401 miRNAs presenting a combination of both (21\%) while, on the other hand, $290(15.2 \%)$ remained in Intg regions and 140 (7.3\%) overlapping IncRNA genes. As expected, the genomic context is associated with the age of miRNAs (Chi square test $=238.25, \mathrm{p}=2.2 \mathrm{e}-16$ ). This association shows that primatespecific miRNAs present a dominance of overlapping TEs in comparison with non-primate miRNAs, with the TE and TE + PC hosting categories being the major contributors across environments. On the other hand, IncRNAs are highly associated with the miRNA context among the non-primate groups, mainly in the group of miRNAs conserved beyond mammals (Supplementary Fig. S2b).

We made use of the miRNA expression levels in 16 different human tissues extracted from Panwar et al. (2017) to study their correlation across groups of conservation. As seen in Fig. 1d, the tissue specificity is higher at lower evolutionary ages, which may indicate the limited expression breadth of young miRNAs. Also, the expression levels were correlated with age, having the more conserved miRNAs an overall higher expression that may probably be due to their consolidated role in regulatory networks (Fig. 1e).

Due to the evolutionary relevance of the miRNA organization in the genome, we revisited the clustering patterns of the miRBase annotations. When studying the closeness between miRNAs, an increment of distances ranging 1-10kb was found (Supplementary Fig. S2c), which indicates a high accumulation of close miRNAs in certain regions. According to this, we defined that two miRNAs belonged to the same cluster when they were located $10 \mathrm{~kb}$ or closer from each other. A total of 100 clusters were identified in the whole genome (Fig. $1 \mathrm{f}$ and Supplementary Fig. S3), represented by 352 miRNA members. Two thirds of these clusters (64) were constituted only by two genes, while 36 clusters presented more than two miRNAs. Two main clustering hotspots were observed in the chromosomes 14 (42) and 19 (46), as previously reported (Muiños-Gimeno et al. 2010; Guo et al. 2014), while the X chromosome presented a similar amount of clustered miRNAs (57) but more widespread in smaller groups (Fig. 1f). A total of 1552 miRNAs were located in isolated regions. We also found a strong correlation between the clustering patterns of miRNAs and groups of conservation (Fig. 1g). The more conserved miRNAs tend to be found in clusters rather than in isolated regions, something likely related to the conserved role of clustered miRNAs in similar biological processes (Berezikov 2011; Wang et al. 2016).

\section{Nucleotide diversity of miRNAs is strongly shaped by their age, genomic context, and localization}

The genetic variation of the miRNA dataset was analysed in the different miRNA functional regions including a region of the same size on both sides of the precursor ( $5^{\prime}$ and $3^{\prime}$ flanking regions) using 
human genetic variation from the 1000 Genomes project (Fig. 1a; Auton A et al. 2015). A total of 1994 single nucleotide polymorphisms (SNPs) were found in 1025 out of 1904 miRNA regions (53.8\%). From them, 623 SNPs (31.2\%) were located in 466 miRNA precursors (24.5\%), from which 212 SNPs $(10.6 \%)$ were located in 194 mature sequences, and 79 SNPs (4\%) were located only in the seed region of 75 miRNAs. However, when considering only the flanking miRNA regions, twice as long as the region occupied by miRNA precursors, 1371 SNPs (68.8\%) were found in 559 miRNA flanking regions. Therefore, more than half of the variability found in our miRNA regions comes from the flanking regions.

To study the sequence variation of human miRNAs we analysed the nucleotide diversity of 1904 miRNA precursor sequences described in miRBase in the pooled population sample from the 1000 Genomes project. The genomic context refers to the environment where miRNAs originally emerged, which might be determinant to their level of variation. We calculated the global nucleotide diversity $(\mathrm{Pi})$ in the whole precursor sequence by considering the age, location, and clustering of the miRNAs (Fig. 2). We found significant differences when comparing the Pi of miRNAs in the different contexts (Kruskal-Wallis $p=$ 0.013). Fig. 2a shows that miRNAs harboured by TEs exhibit a significantly higher $\mathrm{Pi}$ than in other genomic contexts. Next, we examined the TE-family specific diversity of the hosted miRNAs and wondered which TE families contribute more to this high diversity (Supplementary Fig. S4). We performed a multiple linear regression analysis with the different families as predictors and found that Alu and ERVL are significantly associated with the increase of nucleotide diversity (Alu, $p=0.013$; ERVL, $p=5.1 e-04$ ).

As expected, the evolutionary age is another determinant factor in the miRNA sequence diversity. We found that Pi presents a clear correlation with the miRNA conservation (Fig. 2b), with significant differences among the different groups (Kruskal-Wallis $p=2.4 \mathrm{e}-11$ ). The highest diversity was seen in the miRNAs classified as primate specific (group 1) and the lowest in those conserved beyond mammals (group 4).

Regarding the clustering patterns of miRNAs, we found that diversity differences between clustered and isolated miRNAs reached significant levels (Wilcoxon $p=3.7 e-10$ ) (Fig. 2c) which, as seen before, it might be a reflection of the higher conservation of clusters due to their functionality in cooperative processes (Wang et al. 2016; Kabekkodu et al. 2018) and also the fact that most of the clustered miRNAs have originated after common duplication events (Hertel et al. 2006).

Considering the above, sequence diversity levels of human miRNAs seem to be driven by their location, age, and genomic context. These factors might also determine the presence of mutations in miRNA sequences that could affect their expression, hairpin folding and even their ability to bind their target genes and, therefore, be determinant for their evolutionary trajectory. Because of that, we wanted to study the integrated contribution of all these factors to the observed diversity differences. We applied a multiple linear regression model to the diversity data and the different miRNA categories (genomic context, evolutionary age, and clustering). The regression model showed that age (being primate specific, $p=3.3 e-$ 03 ), clustering (being isolated, $p=3.6 e-04$ ) and genomic context (not being intergenic, $p=0.015$ ) are predictors significantly associated with the increase of $\mathrm{Pi}$ in human miRNAs. 


\section{An excess of diversity in the seed region is driven by a reduced number of miRNAs}

The analysis of the nucleotide diversity (Pi) across different miRNA regions indicated an overall higher diversity in the precursor and flanking regions compared to the rest of regions (Wilcoxon test $p<0.05$ ). Surprisingly, the loop region presented the lowest diversity of the whole miRNA hairpin (Fig. 2d). This might reflect the importance of this region in the hairpin folding, which is determinant for the processing of the primary sequence. Previous studies (Torruella-Loran et al. 2016) showed that the seed is the most conserved region of the miRNA, which has been associated with its functional relevance due its central role in target binding. However, our results showed a higher $\mathrm{Pi}$ in the seed than in other conserved regions, like the mature (outside seed) and the loop (Wilcoxon pairwise comparisons $p=1.1 \mathrm{e}-03$ and $p=5.6 \mathrm{e}-03$, respectively). It is worth noting that this level of diversity in the seed comes from the variation of a small set of miRNAs $(75,2.9 \%)$, showing that, indeed, most of the human miRNAs are conserved in their seed. On the other hand, the seed region presented values of SNP density similar to those in the mature outside the seed (Fig. 2e), which suggests that, considering the values of nucleotide diversity, the seed region is more populated by high frequency variants than the mature region. The region-specific levels of diversity were studied in the whole range of minor allele frequency (MAF), where the seed region was consistently found with diversity levels below the mature region until a frequency $\sim 50 \%$ (Fig. 2f). This shows that no bias in the variant content is confounding these results. Overall, these data suggest that the high diversity observed in this set of miRNAs might be a consequence of the specific targeting of positive selection processes, as discussed below.

Previous reports on miRNA targeting (Grimson et al. 2007; Wheeler et al. 2009) show that not only the seed region but also certain positions in the mature sequence are involved in target binding. To further analyse the variation in the miRNAs, nucleotide diversity was studied at position basis in the whole precursor sequence (Fig. $2 \mathrm{~g}$ ). As expected, the general pattern shows that the mature sequences are located in a valley of diversity, which confirms their overall conservation. Different levels of diversity are seen in the mature sequence (Fig. 2g). More specifically a decrease in diversity is seen at the $3^{\prime}$ end, corresponding to the region known as participating in the complementary binding of mRNAs.

\section{Highly differentiated miRNA SNPs are enriched in signals of positive selection, expression variation and disease}

The excess of diversity found in the seed region may respond to particular processes of positive selection that generate frequency shifts at population level. These population-specific changes could affect the miRNA binding to the target gene and change the targeting profiles. In this line, we wanted to study the population-specific patterns of diversity found within the miRNA seed regions. In Supplementary Fig. S5 we show the Pi values of the seed regions from a total of 60 miRNAs presenting genetic variants (DAF >= $5 \%$ ) calculated in each of the 26 populations of our study. The clustering pattern of diversity sharing among populations reflects the similarities of demographic and potential evolutionary histories in the same continental group. As expected, African populations (AFR) are clustered separately from the other populations, showing the highest differentiation probably due to the Out-of-Africa event. A higher diversity 
sharing is seen among the non-African populations. There are some clear continental-specific groups of miRNAs that might be the result of demographic dynamics and/or genetic drift, but also of local processes of positive selection on certain alleles. Considering the group-specific membership of miRNA alleles we found that $37 \%$ (22) are exclusively present in AFR, while $13 \%$ (8) are found in non-Africans, private or shared among other groups (European (EUR), American (AMR), EastAsian (EAS) and South Asian (SAS)). The other alleles are shared between African and non-African populations $(50 \%, 30)$, being $21(35 \%)$ present in all continents.

Next, mean population differentiation $\left(\mathrm{F}_{\mathrm{st}}\right)$ values across all possible population comparisons were calculated for the different miRNA regions (Fig. 3a). As shown, the seed presents an overall $\mathrm{F}_{\mathrm{st}}$ score higher than the rest of the mature sequence in almost all the compared groups. This tendency is stronger in comparisons including AFR populations than non-African ones. Although demographic dynamics are generally the main cause in the existing differentiation between populations, the high $\mathrm{F}_{\mathrm{st}}$ values in the seed, compared to other conserved regions like the mature (outside seed) and the loop, suggest that this region could have been particularly targeted by processes of positive selection. Surprisingly, in contrast with the overall low diversity values seen before, the loop region also exhibits particularly high $\mathrm{F}_{\mathrm{st}}$ scores in some comparisons, especially in the AFR vs SAS populations.

Further, we evaluated the potential functionality of the precursor region-specific SNPs by contrasting their overall Combined Annotation Dependent Depletion (CADD) score distributions, a statistic designed to measure the deleteriousness of human variants (Rentzsch et al. 2019). As shown in Fig. 3b, the CADD scores associated with the loop and seed regions are slightly higher than the rest of the precursor sequence, although non-significant. This evidence reinforces the idea that these regions are specifically implicated in processes potentially involved in adaptive selection.

We wanted to examine the extent to which the top $F_{\text {st }}$ scoring SNPs participate in putative signatures of recent positive selection. We focused on signals characterized by the presence of long haplotypes at high (ongoing hard sweeps) and moderate frequencies (soft sweeps) in individual populations, detected by the statistics integrated haplotype score (iHS) (Voight et al. 2006) and the number of segregating sites by length (nSL) (Ferrer-Admetlla et al. 2014) (see Methods). We pooled the SNP set (100,16\%) that showed extreme Fst values $(>99 \%)$ in the whole miRNA precursor sequence in all population comparisons and explored their involvement in selective sweeps. Among these top SNPs we found that $23 \%$ and $18 \%$ present extreme iHS and nSL scores ( $\geq 2)$, respectively, in at least one population, while the proportion of highly scoring SNPs in the whole dataset is only $13.8 \%$ (iHS) and $11.5 \%(\mathrm{nSL})$. This result suggests that highly differentiated SNPs in miRNAs are more likely to be found in genomic regions that hold signatures consistent with recent positive selection (iHS Chi square test $=11.29, p=7.8 \mathrm{e}-04$; $\mathrm{nSL}$ Chi square test $=$ $6.74, p=9.4 e-03)$.

Nucleotide changes in regions involved in miRNA sequence processing (pre, loop) and target binding (mature, seed) might affect the regulation of their target genes and, therefore, generate expression variation that could be related to genetic disorders, but also to phenotypic adaptations. Thus, we used the 
Genotype-Tissue Expression (GTEx) Project catalog (v7) of associated eQTL-eGene pairs to study the potential impact of our miRNA-harbouring top SNPs in gene expression variation (Aguet F et al. 2017). Among the top 100 SNPs in the precursor sequences, 54\% (54) are reported as significant expression Quantitative Trait Loci (eQTLs) by GTEx, while the 24.7\% (154) are found in the whole dataset of 623 SNP located in miRNA precursors. Also, we used the most recent release of the genome-wide association studies (GWAS) catalog (v1.0) (Buniello et al. 2019) to evaluate the extent to which these highly differentiated SNPs are associated with genetic diseases and traits. In this case, 5\% (5) of the top SNPs present significant associations in GWAS studies, while only $1.8 \%$ (11) were found associated in the whole SNP dataset. These results indicate that highly differentiated miRNA-harbouring SNPs are more likely to be reported as significant eQTLs (Chi-square test $=33.994, p=5.5 e-09$ ) and GWAS associated SNPs (Chi square test $=6.7841, p=9.2 \mathrm{e}-03$ ), which suggests their implication in expression variation and human diseases.

\section{miRNA recent evolution might be driven by targeted processes in their seed related to positive selection and disease}

In order to identify potential candidate miRNAs under the selection pressures of local adaptations, we calculated mean $F_{\text {st }}$ values in the whole mature sequence. Fig. 3c shows the genome wide distribution of mature-specific $F_{\text {st }}$ values in the three comparisons of reference (Utah Europeans (CEU) vs Han Chinese (CHB), CEU vs Yoruba (YRI) and CHB vs YRI), where three miRNAs are found in the top 1\% (hsa-miR1269b, hsa-miR-412-3p, hsa-miR-4707-3p) and 22 above the 5\% (Table 1). Surprisingly the three most divergent miRNAs belong to conservation groups older than primate specific, which suggests that these population-specific changes might respond to potential adaptations that affect well-established regulatory pathways. These top candidate miRNAs harbour 10 SNPs within their seed regions (10 miRNAs) and 14 SNPs in other positions of the mature sequence (14 miRNAs). As seen in Fig. 3d, seedharboring SNPs like rs2273626 (hsa-miR-4707-3p) present the most extreme $F_{\text {st }}$ scores in the candidate mature sequences and reach top values (>99.98\%) in the whole miRNA distribution. Among these, seven SNPs in both seed (rs6771809, rs77651740, rs28655823, rs2273626, rs2168518, rs7210937, rs3745198) and mature regions (rs56790095, rs73239138, rs404337, rs2155248, rs61992671, rs12451747, rs73410309) were reported by GTEx as significantly associated to gene expression variation.

As seen before, the presence of SNPs in the seed region might lead to variations of the miRNA targeting profiles. In order to evaluate the degree of change that a single SNP might generate, we adapted the TargetScanHuman (Agarwal et al. 2015) pipeline to predict the allele-specific targets of the seed-variant candidates. When comparing the sets of target genes due to the ancestral and derived alleles we observed that, among the top ten miRNAs with SNPs in their seed, only two present a cosine similarity above 70\% (hsa-miR-10524-5p and hsa-miR-4513), while the other candidates fall below $23 \%$. This indicates the dramatic target shift that a single SNP generates and might be involved in regulatory adaptations (Table 2). 
Next, we wanted to examine these candidate miRNAs with SNPs showing the highest population differentiation more in depth. We reviewed the literature looking for particular phenotypes in human populations and potential regulatory processes where these variants might have been associated with. Among the ten miRNA candidates with SNPs located in the seed, all except one (hsa-miR-10524-5p) have been related to disease and, specially, with different types of cancers (Table 1), showing some of them differences among populations attributable to genetic risk factors, like breast cancer (BC), colorectal cancer (CRC) and gastric cancer (GC) (Sung et al. 2021). Particularly, three of these miRNAs (hsa-miR4472, hsa-miR-4513 and hsa-miR-6826-5p) were associated with BC, two (hsa-miR-4472 and hsa-miR4741) with CRC and two (hsa-miR-938, hsa-miR-4513) with GC. In four out of the nine miRNAs related to disease the miRNA association was linked to the presence of the variant (rs12416605 in hsa-miR-938, rs7210937 in hsa-miR-1269b, rs2168518 in hsa-miR-4513 and rs2273626 in hsa-miR-4707-3p) (Table 1). When considering the 14 miRNA candidates with SNPs located in the mature regions, we observed that, all except one, for which no previous data have been reported (hsa-miR-6811), have been previously related to disease (Table 1). Among the associations with cancers showing differences on their risk among populations, five (hsa-miR-196a-3p, hsa-miR-646, hsa-miR-1269a, hsa-miR-6826-5p and hsa-miR8084) have been associated with $B C$, five (hsa-miR-196a-3p, hsa-miR-646, hsa-miR-1269a, hsa-miR-6071 and hsa-miR-6826-5p) with CRC, and four (hsa-miR-196a-3p, hsa-miR-646, hsa-miR-1269a and hsa-miR1304-3p) with GC. In four out of the 13 miRNAs related to disease the miRNAs association was linked to the presence of the variant (rs11614913 in hsa-miR-196a-3p, rs61992671 in hsa-miR-412-3p, rs6513497 in hsa-miR-646 and rs73239138 in hsa-miR-1269a) (Table 1).

In particular, for rs 11614913 in hsa-miR-196a-3p $\left(F_{s t}=0.24\right)$ the derived T allele has been associated with a decreased risk of different types of cancers, including breast and gastrointestinal cancers, principally in Asian populations. The frequency of the derived T allele is higher in East Asians ( 54\%) than in Europeans (CEU 44\%) and remarkably higher than in Africans ( $13 \%$ ), which may explain differences in the presentation of these types of cancer among populations and would agree with selective processes in this SNP. Similarly, for rs12416605 in hsa-miR-938 $\left(F_{\text {st }}=0.21\right)$, the derived T allele has been reported as a protective factor for the susceptibility to suffer a diffuse subtype of GC with the finding of a higher frequency of the T allele in Europeans compared with Asians ( 29\% vs. 2\%), which would agree with the reported higher predisposition to GC in Asian populations (Torruella-Loran et al. 2019). In this regard, also the T allele of rs73239138 in hsa-miR-1269a $\left(F_{s t}=0.22\right)$ has been significantly associated with a decreased risk of GC in a Chinese population (Table 1).

Although most of the literature is centered on cancer diseases, other pathologies showing population differences worldwide have been linked to some of these miRNA candidates and SNPs. The T allele of rs11614913 in hsa-miR-196a-3p (highest frequency in Asian populations: 54\%) shows a pleiotropic effect being not only associated with cancer but also with the risk of developing coronary artery disease (CAD) (Fragoso et al. 2019), as well as the T allele of rs2168518 in hsa-miR-4513 (highest frequency in European populations: $61 \%$ ), which has been strongly associated with increased susceptibility to CAD and other related pathologies and physiological states showing risk differences among populations such 
as glucose homeostasis, blood pressure, and age-related macular degeneration (Mir et al. 2019; Ghanbari et al. 2014 and 2017; Li et al. 2015).

Additionally, among the candidate SNPs with the highest $F_{\text {st }}$ scores in the top $1 \%$ is rs $2273626\left(F_{s t}=\right.$ 0.57), located in the seed region of hsa-miR-4707-3p. A neuroprotective role for the derived T allele in the progression of glaucoma has been reported (Ghanbari et al. 2017), which goes in line with the negative association of rs2273626 with the disease (Springelkamp et al. 2017). This SNP shows a derived allele frequency of $\sim 3 \%$ in African populations and more than $50 \%$ in non-Africans (Fig. $4 \mathrm{a}$ ), which would agree with the higher incidence of glaucoma in Africans (Abu-Amero et al. 2015). Remarkably, the extended haplotype homozygosity $(\mathrm{EHH})$ decay on this variant indicates the presence of longer haplotypes harbouring the derived allele in non-African populations (Fig. 4b), which is consistent with the occurrence of positive selection processes favouring the neuroprotective allele since the Out-of-Africa event.

\section{Discussion}

The increasing discovery of naturally occurring variation in the human genome, together with the improvement in annotation strategies of non-protein coding genes, have made it possible to study the potential consequences of mutations in the human miRNAs. As a dense layer of post-transcriptional regulation, miRNAs are expected to be highly susceptible to the occurrence of mutations in their sequences. However, in this analysis, along with previous studies (Quach et al. 2009; Carbonell et al. 2012), we discuss the unexpected level of variation in the critical regions of these regulatory molecules and its possible relationship with evolutionary processes associated with disease.

We implemented a computational pipeline to annotate and analyse the nucleotide diversity and selection signatures of the most updated catalog of genetic variation from 1000 Genomes Project (phase III), in the most complete collection of annotated human miRNAs to date (miRBase, v.22). We integrated the analysis of miRNA variation with a sophisticated software for target prediction, TargetScanHuman, which was adapted to identify allele-specific target genes in seed-harbouring SNP miRNAs. This method, unlike others previously published (Riffo-Campos et al. 2016), incorporates multiple features from target conservation to sequence context to generate more accurate prediction scores. As a result, we provided a robust approach to compare the allele-driven targeting and estimate the extent of the shift generated in the target gene profiles of seed-harbouring SNP miRNAs. We also integrated novedous statistical methods sensitive to different modes of selective sweeps (hard and soft) to capture a wider range of selection signatures than previously reported for human miRNAs.

Until now very few studies have considered the integrated role of the different genomic factors that might have shaped the global diversity of the human microRNAome (Gallego et al. 2016). Here we show that the expansion of new miRNAs in the primate lineage, their location in the genome and the role of hosting TEs are significantly associated with the increase in miRNA diversity, something that might be related with the evolutionary boost of the miRNA system in the human genome. Furthermore, against the common belief, here we report a global excess of variation in the seed, which appears as the most diverse 
region among the traditionally conserved functional regions of miRNAs. This is in contrast with the low diversity found in the loop, which evidences the evolutionary constraints due to its role in hairpin folding. These signals stresses the importance of the secondary structure in maintaining the stability of the RNA molecule and determining the balance between miRNA biogenesis, particularly the binding of the miRNA with the Drosha-DGCR8 complex, and miRNA turnover (Han et al. 2006; Guo et al. 2015). Moreover, the population differences found in these two regions are among the highest in the whole precursor sequence, something compatible with targeted evolutionary-driven processes that might be implicated in regulatory advantages. These processes were evaluated in the present study by identifying a global enrichment in positive selection signals (selective sweeps) among the highest differentiated SNPs across populations, showing the potential of these miRNAs and their regulatory networks to drive populationspecific adaptations in agreement with some previously reported works (Quach et al. 2009; Li et al. 2012; Torruella-Loran et al. 2016).

Either by changing their targeting profiles or modifying their expression levels, it is clear that miRNA networks are more versatile to sequence changes than reported until now. We show that a significant fraction of human miRNAs may participate in gene expression variation driven by the presence of eQTLs in their sequences. This could be related to the regulatory plasticity that miRNAs have proven to hold and that might be determinant in adaptive changes at regulatory level. However, the phenotypic consequences of adaptive changes in these molecules are far to be properly understood. The great target breadth of miRNAs and the massive complexity of their regulatory networks make changes in their sequences affect multiple pathways simultaneously. Therefore, selective forces that rewire these networks might also be behind population-specific susceptibilities to different disorders. In this line, here we show that human miRNAs are also enriched in variants associated with specific human traits and diseases reported by GWAS studies and provide a collection of miRNA alleles that were reported to affect individuals differently depending on their genetic ancestries.

In this regard, some of the miRNAs with SNPs showing the highest population differentiation have been found associated with diseases that show different population prevalence worldwide. One of the clearest examples is the case of rs 12416605 in hsa-miR-938, whose derived T allele has been reported to confer protection against the diffuse subtype of gastric cancer (GC) through one of its targets, the chemokine CXCL 12 (Torruella-Loran et al. 2019), reported as playing a critical role in cell migration and invasion (Izumi et al. 2016). This cancer seems to be promoted by the amplified repression of CXCL 12 specifically mediated by the rs 12416605 ancestral C allele (Torruella-Loran et al. 2019), which makes C-allele carriers more susceptible to develop GC metastasis. This would be in agreement with the finding of a higher frequency of the T allele in Europeans ( 29\%) compared with Asians ( 2\%), which is reflected by a highglobal fixation index $\left(\mathrm{F}_{\mathrm{st}}\right)$, and may influence the existing geographical clinical differences in $\mathrm{GC}$ between Asian and non-Asian populations (Lin et al. 2015).

Among non-cancer diseases we found the T alleles of rs11614913 in hsa-miR-196a-3p and rs2168518 in hsa-miR-4513 associated with increased susceptibility to coronary artery disease (CAD). Although this disease seems to be highly dependent on environmental factors, with over $60 \%$ of current cases occurring 
in developing countries (Beltrame et al. 2012), population differences in CAD susceptibility are envisaged. In that context, the most striking finding is for primary open-angle glaucoma (POAG), a complex neurodegenerative disorder, dependent on environmental and genetic factors, that causes irreversible blindness and affects approximately 70 million people worldwide. Recent studies report a highly biased prevalence of the disease towards individuals with African ancestry, followed by Asians and Europeans (Abu-Amero et al. 2015). Several genes have been found associated with the progression of POAG by diverse GWAS studies. Among them, the caspase recruitment domain family member 10 (CARD10) seems to confer a neuroprotective role by increasing the survival and proliferation of retinal ganglion cells (Khor et al. 2011), whose apoptosis is enhanced in POAG. In Ghanbari et al. (2017), the authors demonstrated by allele-specific in vitro validation that the miR-4707-3p rs2273626 derived T-allele generates a lower repression of CARD10. A weaker binding to the target seems to be behind this expression change, which we further validated with TargetScanHuman, reporting a greater repression score by the ancestral allele (0.632) than the derived allele (0.124). The authors propose that the neuroprotective role of $C A R D 10$ in the progression of glaucoma is associated with this lower repression, supported by the negative association of rs2273626 with the disease (Springelkamp et al. 2017). We suggest that the allele-specific regulation of CARD10 through hsa-miR-4707-3p might contribute to the population disparities prevalence of POAG and that this differential regulation is driven by processes of positive selection that promote the neuroprotective role of rs2273626 derived T-allele in non-African populations.

In conclusion we show that, despite the strong selective pressures that maintain miRNA conservation, several miRNA variants might have suffered the effect of positive selection and may account for phenotypic diversity among human populations being, in some cases, related to disease. Even though we identify some of these miRNA variants and, in certain cases, functional data shows allele-specific regulation of specific target genes, the extent to which most of these miRNA mutations contribute to differences in disease risk among populations remains to be investigated. One of the main limitations of the analysis of positive selection in miRNAs is their small size. Haplotype-based statistics like iHS and $\mathrm{nSL}$ rely on the detection of long unbroken haplotypes that might span thousands of base pairs on both sides of the selected locus, which hinder the identification of the true target of selection. The intronic origin of a substantial number of human miRNAs also makes difficult the identification of the causal genomic locus of the selection signature, potentially being originated either by the miRNA or the hosting gene. The conclusive evidence to understand the contribution of miRNAs to the recent evolutionary history of humans is the experimental validation of the genotype-phenotype association. However, the multiple potential targets of miRNAs and the side effects generated by sequence changes in the nonselected cellular processes makes this validation a difficult task. New methods and more data are needed to continue filling this gap between the genetic change and the phenotypic adaptation.

\section{Materials And Methods}

\section{Human miRNA coordinates and functional region annotation}


The human miRNA genomic coordinates were downloaded from the last release of the miRBase annotation database (v.22, March 2018) (Kozomara et al. 2019, http://www.mirbase.org/). This dataset contains the coordinates of 1918 human miRNA precursor transcripts and their mature sequences that were converted to hg19 genome assembly with liftOver (Hinrichs et al. 2006). From this conversion, four miRNA genes were dropped from the original dataset, and 10 were not able to be located in any chromosome, being also removed and leaving a total of 1904 precursor sequences. A custom script was designed to extract the individual functional regions of each miRNA. As shown in Fig. 1a we differentiated the "seed" region (positions 2-8), the mature ("mat") region outside the seed, the "loop" (region between two mature sequences) and the precursor regions ( $5^{\prime}$ and 3 ' sides) outside the mature and loop. We also considered precursor flanking regions on both sides ( $5^{\prime}$ and $\left.3^{\prime}\right)$ of each miRNA hairpin, having the same length as the whole precursor sequences. An additional category was created in order to accommodate the regions that overlap between different miRNAs ("ovlp"), these miRNAs were treated differently due to the difficulty of analysing the overlapping regions. In the analysis of region-specific diversity the miRNAs with "ovlp" regions (71) were discarded. A different degree of mature annotation was seen in the miRBase transcripts: 959 transcripts out of the 1904 (50.3\%) presented both mature sequences annotated ( $5 p$ and $3 p$ arms), allowing to completely describe the different regions of the precursor sequences. However, in 945 transcripts (49.7\%) only one mature sequence was reported. In these cases, the description of the whole precursor sequence was limited to the boundaries of the single mature described (the specific boundaries of the loop region are not able to be defined). Therefore, when extracting the functional regions of the miRNA genes, the precursor region was considered as the whole portion that encompasses from the end of the given mature sequence to the start of the opposite flanking region (this would retain as "precursor" the "loop" region, the unannotated mature region, and the actual premature region of that arm). The "loop" region was only extracted when the two mature sequence coordinates were given. These inconsistencies in the annotation of the miRNA transcripts were taken into account throughout the analysis (Fig. 1a).

\section{Computational analyses of genomic context, evolutionary age, and clustering annotation}

A computational pipeline was used to integrate the tools to annotate miRNAs, locate variants in the miRNA sequences and perform the statistical calculations for the analysis of diversity, positive selection, and target prediction. This pipeline was adapted to work in a high performance computing (HPC) environment based on the cluster management and job scheduling system SLURM. In order to obtain the genomic context of miRNAs, we intersected the GENCODE 19 of PC genes and IncRNA genes annotations (v.29) (Frankish et al. 2019) with the miRNA coordinates with the multipurpose software Bedtools (Quinlan et al. 2010), which allowed us to find coordinate overlaps between two or more sets of genomic regions with a minimum overlap of $1 \mathrm{bp}$ (Bedtools intersect functionality). The RepeatMasker open-4.0.5 database (repeat library 20140131) (Smit et al. 2013-2015), which looks for interspersed repeats and lowcomplexity DNA (simple repeats, microsatelites), was also used to define the overlap of miRNAs with repetitive elements. miRNAs were classified based on their evolutionary age by merging the classifications obtained in Iwama et al. (2013) and Santpere et al. (2016). We grouped the miRNAs in the following categories: primate-specific (group 1, previous 5 to 12 groups in Iwama et al. 2013); eutherians 
(group 2, previous 1 to 4); metatheria and prototheria (group 3, previous -1 to 0 ) and conserved beyond mammals (group 4, previous -2 to -3). The remaining 281 miRNAs were non-classified due to absence of data or discrepancies between the two studies in their evolutionary age. In order to obtain the miRNA clusters, a python-based custom script was designed to calculate the closest distance of each miRNA to any other in the same strand and chromosome. We defined miRNA clusters as groups of two or more miRNA genes separated by 10000 bp or less (Guo et al. 2014). The contributions of the genomic context, evolutionary age and clustering to the nucleotide diversity were obtained by applying a multiple linear regression model $(I m)$, which is based on the programming language R (R Core Team 2020) and seeks to estimate the relationships between these factors (predictors) and the response variable (diversity).

\section{miRNA genetic variation and nucleotide diversity}

Human variation data from The 1000 Genomes project (phase III) (Auton A et al. 2015) was used to annotate the human miRNA dataset. 26 different human populations accounting for a total of 2504 individuals were considered in the analysis, including the admixed populations from South Asia (SAS) and the Americas (AMR). We used the last version of the program BCFtools (v.1.11) (Danecek et al. 2021), for processing and analysing high-throughput sequencing data, to extract the variants located within the miRNA sequences. Only biallelic SNPs with a MAF greater or equal than $1 \%$ in individual populations and $0.5 \%$ in the global population were considered. Unnamed variants were kept and corrected by using the physical position preceded by "rs_" as provisional SNP ID. When computing the derived allele frequency and haplotype-based statistics, the human ancestral alleles annotated in the original VCF files were used to format the REF and ALT fields and the corresponding genotypes of the individuals. Any SNP whose ancestral status was unknown or did not match with the reference or alternative alleles were removed from the dataset. The overall pairwise mismatches per SNP (pi) were calculated with BCFtools in the whole miRNA SNP dataset, after that the nucleotide diversity $(\mathrm{Pi})$ per region was computed by obtaining the diversity per nucleotide in the whole length $(L)$ of each functional region $(\mathrm{Pi}=\mathrm{pi} / \mathrm{L})$. We considered each region category (flank, pre, mat, seed, loop) as a single sequence instead of calculating the nucleotide diversity in the regions of the individual miRNAs. The nucleotide diversity per position was calculated by aligning the precursor transcripts of the whole miRNA dataset and obtaining the mean pi value at each site. In this analysis, the "ovlp" regions were not considered due to the difficulty of interpreting the diversity properties of such overlaps.

\section{Pathogenicity and disease associations of miRNA variants}

The catalog of Combined Annotation Dependent Depletion (CADD) scores (Rentzsch et al. 2019) provides a quantitative way to measure the deleteriousness of single nucleotide polymorphisms (SNPs) in the human genome by prioritizing the functionality and diseases causing variants. This catalog was used to assess the level of pathogenicity of miRNA-harbouring SNPs as a proxy of their functionality. According to Kircher et al. (2014) a threshold of PHRED-scaled CADD score $\geq 10$ is normally used to discern the $1 \%$ most deleterious SNPs of the whole human genome. We also leveraged the GWAS (v1.0) catalog (Buniello et al. 2019) to evaluate the participation of miRNA-harbouring SNPs in human traits. 


\section{Calculation of $\mathrm{F}_{\mathrm{st}}, \mathrm{iHS}$ and $\mathrm{nSL}$ scores}

Population fixation indexes $\left(\mathrm{F}_{\mathrm{st}}\right)$ were computed by using the Hudson estimator of the $\mathrm{F}_{\mathrm{st}}$ statistic, which is not affected by the sample size and does not overestimate the $F_{s t}$ scores in comparison with others (Bhatia et al. 2013), in all the variant miRNAs. The calculations were performed by pairwise comparison between the 26 populations used from the 1000 Genome project dataset. These $F_{\text {st }}$ scores were normalized by frequency performing a linear regression of the estimator values and the global MAF. The residual values were used as the final $\mathrm{F}_{\mathrm{st}}$ scores. We extended the analysis of selection with two haplotype-based statistics: iHS (Voight et al. 2006) and nSL (Ferrer-Admetlla et al. 2014). These tests rely on the detection of blocks of homozygosity by the EHH statistic (Extended Haplotype Homozygosity) introduced by (Sabeti et al. 2002). A recent positive selection signal is found when these blocks present moderately high or intermediate frequency of derived alleles. The iHS test was designed to detect ongoing hard sweep signals, signatures characterized by the presence of a single sweeping haplotype at high frequency in their way to fixation. On the other hand, nSL was designed to detect either ongoing hard and soft sweep signatures with a greater power than iHS. In the case of soft sweeps, these are signatures of selection on standing variation, where more than one haplotype is sweeping at intermediate frequencies. The calculations of iHS and nSL were computed with the software selscan (Szpiech et al. 2014), an application that implements different haplotype-based statistics in a multithreaded framework. We allowed for a maximum gap of $20 \mathrm{~kb}$ and kept only SNPs with a minor allele frequency (MAF) higher than $5 \%$. This statistic is standardized (mean 0 , variance 1 ) by the distribution of observed scores over a range of SNPs with similar derived allele frequencies. The standardization was performed in each population separately by using the norm function, also contained in the selscan package (Voight et al. 2006).

\section{Target predictions}

The program TargetScanHuman (TSH, release 7.2) (Agarwal et al. 2015) was used to perform the miRNA target predictions. The perl-based pipeline used by the authors (http://www.targetscan.org/cgibin/targetscan/data_download.vert72.cgi), together with the ViennaRNA package (Lorenz et al. 2011), were locally implemented and adapted to perform predictions from a custom miRNA dataset. This pipeline was composed by three different steps: (i) target site identification across the set of $3^{\prime} U T R$ regions of the human genome, (ii) the probability of conserved targeting $\left(P_{c t}\right)$ calculations and (iii) the calculations of the context++ scores, which integrates different genomic features implicated in targeting efficiency. miRNA families and species information were downloaded from the targetscan.org Data Download page. In order to calculate the $P_{\text {ct }}$ parameters, the 3'UTR dataset from the GENCODE version 19 (Ensembl 75) was obtained as a 84-way alignment from the same download page. As described in Agarwal et al. (2015), only the longest 3'UTR isoform of each gene was used as representative transcript. To account for the miRNA variation in the target predictions, the variable positions in the miRNA seed regions (ancestral and derived states) were considered and incorporated into the TSH pipeline. Two different miRNA datasets were obtained when accounting for the ancestral and derived alleles of the 
SNPs found in the seed regions. As described in Agarwal et al. (2015), the accumulated weighted-scores per target gene were calculated as the sum of the individual target site weighted-scores, which is the final score associated with each target gene. As suggested by the authors, in order to remove the potential false positives, we applied a custom per-site-based filtering strategy. Since negative weighted scores are associated with mRNA repression, only the per-site weighted scores below zero were considered and, from these, the per-miRNA 50th percentile was used as threshold to obtain the putative true target sites in each miRNA. In order to analyse the overlap between the predicted targets of the derived and ancestral miRNA alleles we used the cosine similarity (Hill et al. 2014), which was calculated by the total number of overlapping genes divided by the square root of the product of the number of targets of both alleles.

\section{Analysis of expression levels and expression variation}

The catalogue of expression Quantitative Trait Loci (eQTLs) provided by the Genotype-Tissue Expression (GTEx) Project (Aguet F et al. 2017) was used to assess the implication of miRNA-harbouring variants in expression variation. Expression data from 16 different human tissues (bladder, blood, brain, breast, hair follicle, liver, lung, nasopharynx, pancreas, placenta, plasma, saliva, semen, serum, sperm, and testis) was taken from Panwar et al. (2017). We used 2085 mature miRNAs from this dataset for which evolutionary age was available. Reads per million (RPM) values were analysed for each mature miRNA separately,

whose conservation status were determined by the precursor molecule following the classification criteria described before. A miRNA was considered to be expressed in a specific tissue when its reads were unequal to zero in at least one sample from that tissue. For the comparative analyses of the expression levels among conservation groups we took the total number of reads in the 16 tissues for all the miRNAs within each group.

\section{Declarations}

\section{Funding}

This study has been possible thanks to the Chilean Agencia Nacional de Investigación y Desarrollo - ANID (FONDECYT regular $n^{\circ} 1170446$ ), the Chilean Ministry of Education (MAG-1995), grant PID2019110933GB-I00/AEI/10.13039/501100011033 awarded by the Spanish Agencia Estatal de Investigación (AEI), Ministerio de Ciencia, Innovación y Universidades (MCIU, Spain), the support of Secretaria d'Universitats i Recerca del Departament d'Economia i Coneixement de la Generalitat de Catalunya (GRC 2017 SGR 702), part of the "Unidad de Excelencia María de Maeztu", funded by the AEI (CEX2018000792-M). P.V-M is supported by an FPI PhD fellowship (FPI-BES-2016-077706) part of the "Unidad de Excelencia María de Maeztu" funded by MINECO (ref: MDM-2014-0370).

\section{Conflicts of interest/Competing interests}

The authors affirm that there is no conflict of interest to declare.

\section{Availability of data and material}


All data and material used in this study is fully available from the source public databases.

\section{Code availability}

\section{Authors' contributions}

YE-P, HL conceived the study. PV-M, AG, HL and YE-P analysed and interpreted the data. PV-M, AG and YE$P$ wrote the manuscript. PV-M, AG, HL, YE-P and JB revised the manuscript. All authors approved the final manuscript.

\section{Ethics approval}

Not applicable.

\section{Consent to participate}

Not applicable.

\section{Consent for publication}

All authors consent the publication of the final manuscript.

\section{References}

Abu-Amero K, Kondkar AA, Chalam K V. (2015) An updated review on the genetics of primary open angle glaucoma. Int. J. Mol. Sci. 16:28886-28911. https://doi.org/10.3390/ijms161226135

Agarwal V, Bell GW, Nam JW, Bartel DP (2015) Predicting effective microRNA target sites in mammalian mRNAs. Elife 4:. https://doi.org/10.7554/eLife.05005

Aguet F, Brown AA, Castel SE, et al (2017) Genetic effects on gene expression across human tissues. Nature 550:204-213. https://doi.org/10.1038/nature24277

Ahmad M, Shah AA (2020) Predictive role of single nucleotide polymorphism (rs11614913) in the development of breast cancer in Pakistani population. Per Med 17:213-227. https://doi.org/10.2217/pme-2019-0086

Arisawa T, Tahara T, Shiroeda H, et al (2012) Genetic polymorphisms of IL17A and pri-microRNA-938, targeting IL17A 3'-UTR, influence susceptibility to gastric cancer. Hum Immunol 73:747-752. https://doi.org/10.1016/j.humimm.2012.04.011

Auton A, Abecasis GR, Altshuler DM, et al (2015) A global reference for human genetic variation. Nature 526:68-74

Bartel DP (2018) Metazoan MicroRNAs. Cell 173:20-51. https://doi.org/10.1016/j.cell.2018.03.006 
Bartel DP (2009) MicroRNAs: Target Recognition and Regulatory Functions. Cell 136:215-233. https://doi.org/10.1016/j.cell.2009.01.002

Beecham GW, Dickson DW, Scott WK, et al (2015) PARK10 is a major locus for sporadic neuropathologically confirmed Parkinson disease. Neurology 84:972-980.

https://doi.org/10.1212/WNL.0000000000001332

Berezikov E (2011) Evolution of microRNA diversity and regulation in animals. Nat. Rev. Genet. 12:846860

Bhatia G, Patterson N, Sankararaman S, Price AL (2013) Estimating and interpreting FST: The impact of rare variants. Genome Res 23:1514-1521. https://doi.org/10.1101/gr.154831.113

Bi Y, Guo S, Xu X, et al (2020) Decreased ZNF750 promotes angiogenesis in a paracrine manner via activating DANCR/miR-4707-3p/FOXC2 axis in esophageal squamous cell carcinoma. Cell Death Dis 11:. https://doi.org/10.1038/s41419-020-2492-2

Borel C, Deutsch S, Letourneau A, et al (2011) Identification of cis- and trans-regulatory variation modulating microRNA expression levels in human fibroblasts. Genome Res 21:68-73.

https://doi.org/10.1101/gr.109371.110

Bu P, Wang L, Chen KY, et al (2015) miR-1269 promotes metastasis and forms a positive feedback loop with TGF- $\beta$. Nat Commun 6:. https://doi.org/10.1038/ncomms7879

Buniello A, Macarthur JAL, Cerezo M, et al (2019) The NHGRI-EBI GWAS Catalog of published genomewide association studies, targeted arrays and summary statistics 2019. Nucleic Acids Res 47:D1005D1012. https://doi.org/10.1093/nar/gky1120

Cai M, Zhang Y, Ma Y, et al (2015) Association between microRNA-499 polymorphism and gastric cancer risk in Chinese population. Bull Cancer 102:973-978. https://doi.org/10.1016/j.bulcan.2015.09.012

Cammaerts S, Strazisar M, Rijk P De, Del Favero J (2015) Genetic variants in microRNA genes: Impact on microRNA expression, function, and disease. Front. Genet. 6. https://doi.org/10.3389/fgene.2015.00186

Carbonell J, Alloza E, Arce P, et al (2012) A map of human microRNA variation uncovers unexpectedly high levels of variability. Genome Med 4.. https://doi.org/10.1186/gm363

Cattaneo M, Pelosi E, Castelli G, et al (2015) A miRNA signature in human cord blood stem and progenitor cells as potential biomarker of specific acute myeloid leukemia subtypes. J Cell Physiol 230:1770-1780. https://doi.org/10.1002/jcp.24876

Chen HC, Tseng YK, Chi CC, et al (2016) Genetic variants in microRNA-146a ( $>$ > G) and microRNA-1269b $(\mathrm{G}>\mathrm{C})$ are associated with the decreased risk of oral premalignant lesions, oral cancer, and pharyngeal cancer. Arch Oral Biol 72:21-32. https://doi.org/10.1016/j.archoralbio.2016.08.010 
Chong GO, Jeon HS, Han HS, et al (2015) Differential MicroRNA Expression Profiles in Primary and Recurrent Epithelial Ovarian Cancer. Anticancer Res 35(5):2611-2617.

Choupani J, Nariman-Saleh-Fam Z, Saadatian Z, et al (2019) Association of mir-196a-2 rs11614913 and mir-149 rs2292832 polymorphisms with risk of cancer: An updated meta-analysis. Front. Genet. 10. https://doi.org/10.3389/fgene.2019.00186

Chuong EB, Elde NC, Feschotte C (2017) Regulatory activities of transposable elements: From conflicts to benefits. Nat. Rev. Genet. 18:71-86

Cojocneanu R, Braicu C, Raduly L, et al (2020) Plasma and tissue specific miRNA expression pattern and functional analysis associated to colorectal cancer patients. Cancers (Basel) 12:.

https://doi.org/10.3390/cancers12040843

Dai H, Hou K, Cai Z, et al (2017) Low-level miR-646 in colorectal cancer inhibits cell proliferation and migration by targeting NOB1 expression. Oncol Lett 14:6708-6714.

https://doi.org/10.3892/ol.2017.7032

Danková Z, Grendár M, Dvorská D, et al. (2020) miRNA profile of luminal breast cancer subtypes in Slovak women. Ceska Gynekol 85(3):174-180.

Danecek P, Bonfield JK, Liddle J, et al (2021) Twelve years of SAMtools and BCFtools. Gigascience 10: https://doi.org/10.1093/gigascience/giab008

Darvishi N, Rahimi K, Mansouri K, et al (2020) MiR-646 prevents proliferation and progression of human breast cancer cell lines by suppressing HDAC2 expression. Mol Cell Probes 53:.

https://doi.org/10.1016/j.mcp.2020.101649

Delić D, Eisele C, Schmid R, et al (2016) Urinary exosomal miRNA signature in type II diabetic nephropathy patients. PLoS One 11:. https://doi.org/10.1371/journal.pone.0150154

Ding H, Shi Y, Liu X, Qiu A (2019) MicroRNA-4513 Promotes Gastric Cancer Cell Proliferation and Epithelial-Mesenchymal Transition Through Targeting KAT6B. Hum Gene Ther Clin Dev 30:142-148. https://doi.org/10.1089/humc.2019.094

Dong L, Deng J, Sun ZM, et al (2015) Interference with the $\beta$-catenin gene in gastric cancer induces changes to the miRNA expression profile. Tumor Biol 36:6973-6983. https://doi.org/10.1007/s13277015-3415-1

Fadhil RS, Wei MQ, Nikolarakos D, et al (2020) Salivary microRNA miR-let-7a-5p and miR-3928 could be used as potential diagnostic bio-markers for head and neck squamous cell carcinoma. PLoS One 15:. https://doi.org/10.1371/journal.pone.0221779 
Fernandez N, Cordiner RA, Young RS, et al (2017) Genetic variation and RNA structure regulate microRNA biogenesis. Nat Commun 8:. https://doi.org/10.1038/ncomms15114

Ferrer-Admetlla A, Liang M, Korneliussen T, Nielsen R (2014) On detecting incomplete soft or hard selective sweeps using haplotype structure. Mol Biol Evol 31:1275-1291.

https://doi.org/10.1093/molbev/msu077

Feschotte C (2008) Transposable elements and the evolution of regulatory networks. Nat. Rev. Genet. 9:397-405

Fragoso JM, Ramírez-Bello J, Martínez-Ríos MA, et al (2019) miR-196a2 (rs11614913) polymorphism is associated with coronary artery disease, but not with in-stent coronary restenosis. Inflamm Res 68:215221. https://doi.org/10.1007/s00011-018-1206-z

França GS, Vibranovski MD, Galante PAF (2016) Host gene constraints and genomic context impact the expression and evolution of human microRNAs. Nat Commun 7:. https://doi.org/10.1038/ncomms11438

Frankish A, Diekhans M, Ferreira AM, et al (2019) GENCODE reference annotation for the human and mouse genomes. Nucleic Acids Res 47:D766-D773. https://doi.org/10.1093/nar/gky955

Friedman RC, Farh KKH, Burge CB, Bartel DP (2009) Most mammalian mRNAs are conserved targets of microRNAs. Genome Res 19:92-105. https://doi.org/10.1101/gr.082701.108

Gallego A, Melé M, Balcells I, et al (2016) Functional Implications of Human-Specific Changes in Great Ape microRNAs. PLoS One 11:. https://doi.org/10.1371/journal.pone.0154194

Gao Y, Ma H, Gao C, et al (2018) Tumor-promoting properties of miR-8084 in breast cancer through enhancing proliferation, suppressing apoptosis and inducing epithelial-mesenchymal transition. J Transl Med 16:. https://doi.org/10.1186/s12967-018-1419-5

Ghanbari M, de Vries PS, de Looper H, et al (2014) A Genetic variant in the seed region of miR-4513 shows pleiotropic effects on lipid and glucose homeostasis, blood pressure, and coronary artery disease. Hum Mutat 35:1524-1531. https://doi.org/10.1002/humu.22706

Ghanbari M, Erkeland SJ, Xu L, et al (2017) Genetic variants in microRNAs and their binding sites within gene 3'UTRs associate with susceptibility to age-related macular degeneration. Hum Mutat 38:827-838. https://doi.org/10.1002/humu.23226

Ghanbari M, Iglesias Al, Springelkamp H, et al (2017) A genome-wide scan for microrna-related genetic variants associated with primary open-angle glaucoma. Investig Ophthalmol Vis Sci 58:5368-5377. https://doi.org/10.1167/iovs.17-22410

Gong J, Tong Y, Zhang HM, et al (2012) Genome-wide identification of SNPs in MicroRNA genes and the SNP effects on MicroRNA target binding and biogenesis. Hum Mutat 33:254-263. 
https://doi.org/10.1002/humu.21641

Grigelioniene G, Suzuki HI, Taylan F, et al (2019) Gain-of-function mutation of microRNA-140 in human skeletal dysplasia. Nat Med 25:583-590. https://doi.org/10.1038/s41591-019-0353-2

Grimson A, Farh KKH, Johnston WK, et al (2007) MicroRNA Targeting Specificity in Mammals:

Determinants beyond Seed Pairing. Mol Cell 27:91-105. https://doi.org/10.1016/j.molcel.2007.06.017

Guo L, Zhao Y, Zhang H, et al (2014) Integrated evolutionary analysis of human miRNA gene clusters and families implicates evolutionary relationships. Gene 534:24-32.

https://doi.org/10.1016/j.gene.2013.10.037

Guo Y, Liu J, Elfenbein SJ, et al (2015) Characterization of the mammalian miRNA turnover landscape. Nucleic Acids Res 43:2326-2341. https://doi.org/10.1093/nar/gkv057

Han J, Lee Y, Yeom KH, et al (2006) Molecular Basis for the Recognition of Primary microRNAs by the Drosha-DGCR8 Complex. Cell 125:887-901. https://doi.org/10.1016/j.cell.2006.03.043

He S, Ou H, Zhao C, Zhang J (2018) Clustering pattern and functional effect of SNPs in human miRNA seed regions. Int J Genomics 2018:. https://doi.org/10.1155/2018/2456076

Heimberg AM, Sempere LF, Moy VN, et al (2008) MicroRNAs and the advent of vertebrate morphological complexity. Proc Natl Acad Sci U S A 105:2946-2950. https://doi.org/10.1073/pnas.0712259105

Hertel J, Lindemeyer M, Missal K, et al (2006) The expansion of the metazoan microRNA repertoire. BMC Genomics 7:. https://doi.org/10.1186/1471-2164-7-25

Hill CG, Jabbari N, Matyunina L V., McDonald JF (2014) Functional and evolutionary significance of human microRNA seed region mutations. PLoS One 9:. https://doi.org/10.1371/journal.pone.0115241

Hinrichs AS, Karolchik D, Baertsch R, et al (2006) The UCSC Genome Browser Database: update 2006. Nucleic Acids Res 34:. https://doi.org/10.1093/nar/gkj144

Ibrahim AA, Ramadan A, Wahby AA, et al (2019) Micro-RNA 196a2 expression and miR-196a2 (rs11614913) polymorphism in T1DM: A pilot study. J Pediatr Endocrinol Metab 32:. https://doi.org/10.1515/jpem-2019-0226

Iwama H, Kato K, Imachi H, et al (2013) Human microRNAs originated from two periods at accelerated rates in mammalian evolution. Mol Biol Evol 30:613-626. https://doi.org/10.1093/molbev/mss262

Izumi D, Ishimoto T, Miyake K, et al (2016) CXCL12/CXCR4 activation by cancer-associated fibroblasts promotes integrin $\beta 1$ clustering and invasiveness in gastric cancer. Int J Cancer 138:1207-1219. https://doi.org/10.1002/ijc.29864 
Ji B, Chen L, Cai Q, et al (2020) Identification of an 8-miRNA signature as a potential prognostic biomarker for glioma. PeerJ 8:. https://doi.org/10.7717/peerj.9943

Jin RH, Yu DJ, Zhong M (2018) MiR-1269a acts as an onco-miRNA in non-small cell Lung cancer via down-regulating SOX6. Eur Rev Med Pharmacol Sci 22:4888-4897.

https://doi.org/10.26355/eurrev_201808_15625

Kabekkodu SP, Shukla V, Varghese VK, et al (2018) Clustered miRNAs and their role in biological functions and diseases. Biol Rev 93:1955-1986. https://doi.org/10.1111/brv.12428

Khor CC, Ramdas WD, Vithana EN, et al (2011) Genome-wide association studies in Asians confirm the involvement of ATOH7 and TGFBR3, and further identify CARD10 as a novel locus influencing optic discarea. Hum Mol Genet 20:1864-1872. https://doi.org/10.1093/hmg/ddr060

Kijima T, Hazama S, Tsunedomi R, et al (2017) MicroRNA-6826 and-6875 in plasma are valuable noninvasive biomarkers that predict the efficacy of vaccine treatment against metastatic colorectal cancer. Oncol Rep 37:23-30. https://doi.org/10.3892/or.2016.5267

Kim HK, Prokunina-Olsson L, Chanock SJ (2012) Common Genetic Variants in miR-1206 (8q24.2) and miR-612 (11q13.3) Affect Biogenesis of Mature miRNA Forms. PLoS One 7:.

https://doi.org/10.1371/journal.pone.0047454

Kircher M, Witten DM, Jain P, et al (2014) A general framework for estimating the relative pathogenicity of human genetic variants. Nat Genet 46:310-315. https://doi.org/10.1038/ng.2892

Kozomara A, Birgaoanu M, Griffiths-Jones S (2019) MiRBase: From microRNA sequences to function. Nucleic Acids Res 47:D155-D162. https://doi.org/10.1093/nar/gky1141

Kurata JS, Lin RJ (2018) MicroRNA-focused CRISPR-Cas9 library screen reveals fitness-associated miRNAs. RNA 24:966-981. https://doi.org/10.1261/rna.066282.118

Lewis BP, Burge CB, Bartel DP (2005) Conserved seed pairing, often flanked by adenosines, indicates that thousands of human genes are microRNA targets. Cell 120:15-20.

https://doi.org/10.1016/j.cell.2004.12.035

Li Q, Chen L, Chen D, Wu X, Chen M (2015) Influence of microRNA-related polymorphisms on clinical outcomes in coronary artery disease. Am J Transl Res 7(2):393-400.

Li JY, Cheng B, Wang XF, et al (2018) Circulating MicroRNA-4739 May Be a Potential Biomarker of Critical Limb Ischemia in Patients with Diabetes. Biomed Res Int 2018:. https://doi.org/10.1155/2018/4232794

Li W, Liu M, Feng Y, et al (2014) Downregulated miR-646 in clear cell renal carcinoma correlated with tumour metastasis by targeting the nin one binding protein (NOB1). Br J Cancer 111:1188-1200. https://doi.org/10.1038/bjc.2014.382 
Li J, Liu Y, Xin X, et al (2012) Evidence for positive selection on a number of microRNA regulatory interactions during recent human evolution. PLoS Genet 8:.

https://doi.org/10.1371/journal.pgen.1002578

Li W, Zhang H, Min P, et al (2017) Downregulated miRNA-1269a variant (rs73239138) decreases the susceptibility to gastric cancer via targeting ZNF70. Oncol Lett 14:6345-6354.

https://doi.org/10.3892/ol.2017.7091

Li Y, Wang YW, Chen X, et al (2020) MicroRNA-4472 Promotes Tumor Proliferation and Aggressiveness in Breast Cancer by Targeting RGMA and Inducing EMT. Clin Breast Cancer 20:e113-e126. https://doi.org/10.1016/j.clbc.2019.08.010

Li Y, Zhu H, Wang J, Qian X, Li N (2019) miR-4513 promotes breast cancer progression through targeting TRIM3. Am J Transl Res 11(4):2431-2438.

Liang X, Lai Y, Wu W, et al (2019) LncRNA-miRNA-mRNA expression variation profile in the urine of calcium oxalate stone patients. BMC Med Genomics 12:57. https://doi.org/10.1186/s12920-019-0502-y

Lin SJ, Gagnon-Bartsch JA, Tan IB, et al (2015) Signatures of tumour immunity distinguish Asian and non-Asian gastric adenocarcinomas. Gut 64:1721-1731. https://doi.org/10.1136/gutjnl-2014-308252

Liu J, Yan J, Zhou C, et al (2015) miR-1285-3p acts as a potential tumor suppressor miRNA via downregulating JUN expression in hepatocellular carcinoma. Tumor Biol 36:219-225. https://doi.org/10.1007/s13277-014-2622-5

Liu Y, He A, Liu B, et al (2018) Rs11614913 polymorphism in miRNA-196a2 and cancer risk: An updated meta-analysis. Onco. Targets. Ther. 11:1121-1139

Lopez-Valenzuela M, Ramírez O, Rosas A, et al (2012) An ancestral miR-1304 allele present in Neanderthals regulates genes involved in enamel formation and could explain dental differences with modern humans. Mol Biol Evol 29(7):1797-1806. doi:10.1093/molbev/mss023

Lorenz R, Bernhart SH, Höner zu Siederdissen C, et al (2011) ViennaRNA Package 2.0. Algorithms Mol Biol 6:. https://doi.org/10.1186/1748-7188-6-26

Lu J, Clark AG (2012) Impact of microRNA regulation on variation in human gene expression. Genome Res 22:1243-1254. https://doi.org/10.1101/gr.132514.111

Lu J, Shen Y, Wu Q, et al (2008) The birth and death of microRNA genes in Drosophila. Nat Genet 40:351355. https://doi.org/10.1038/ng.73

Mao Y, Zou C, Meng F, et al (2018) The SNPs in pre-miRNA are related to the response of capecitabinebased therapy in advanced colon cancer patients. Oncotarget 9:6793-6799.

https://doi.org/10.18632/oncotarget.23190

Page 24/37 
Martin-Guerrero I, Bilbao-Aldaiturriaga N, Gutierrez-Camino A, et al (2018) Variants in the 14q32 miRNA cluster are associated with osteosarcoma risk in the Spanish population. Sci Rep 8:.

https://doi.org/10.1038/s41598-018-33712-4

Min P, Li W, Zeng D, et al (2017) A single nucleotide variant in microRNA-1269a promotes the occurrence and process of hepatocellular carcinoma by targeting to oncogenes SPATS2L and LRP6. Bull Cancer 104:311-320. https://doi.org/10.1016/j.bulcan.2016.11.021

Mir R, Jha C k, Elfaki I, et al (2019) Incidence of MicroR-4513C/T Gene Variability in Coronary Artery Disease - A case-Control Study. Endocrine, Metab Immune Disord - Drug Targets 19:1216-1223. https://doi.org/10.2174/1871530319666190417111940

Muiños-Gimeno M, Guidi M, Kagerbauer B, et al (2009) Allele variants in functional MicroRNA target sites of the neurotrophin-3 receptor gene (NTRK3) as susceptibility factors for anxiety disorders. Hum Mutat. 30(7):1062-1071. doi:10.1002/humu.21005

Muiños-Gimeno M, Montfort M, Bayés M, Estivill X, Espinosa-Parrilla Y (2010) Design and evaluation of a panel of single-nucleotide polymorphisms in microRNA genomic regions for association studies in human disease. Eur J Hum Genet 18(2):218-226. 10.1038/ejhg.2009.165

$\mathrm{Ni}$ Q, Ji A, Yin J, et al (2015) Effects of two common polymorphisms rs2910164 in miR-146a and rs11614913 in miR-196a2 on gastric cancer susceptibility. Gastroenterol. Res. Pract. 2015

Othman N, In LLA, Harikrishna JA, Hasima N (2013) Bcl-xL silencing induces alterations in hsa-miR-608 expression and subsequent cell death in A549 and SK-LU1 human lung adenocarcinoma cells. PLoS One 8.. https://doi.org/10.1371/journal.pone.0081735

Oura K, Fujita K, Morishita A, et al (2019) Serum microRNA-125a-5p as a potential biomarker of HCVassociated hepatocellular carcinoma. Oncol Lett 18:882-890. https://doi.org/10.3892/ol.2019.10385

Pan Y, Chen Y, Ma D, et al (2016) miR-646 is a key negative regulator of EGFR pathway in lung cancer. Exp Lung Res 42:286-295. https://doi.org/10.1080/01902148.2016.1207726

Panwar B, Omenn GS, Guan Y (2017) MiRmine: A database of human miRNA expression profiles. Bioinformatics 33:1554-1560. https://doi.org/10.1093/bioinformatics/btx019

Peng S, Kuang Z, Sheng C, et al (2010) Association of MicroRNA-196a-2 gene polymorphism with gastric cancer risk in a Chinese population. Dig Dis Sci 55:2288-2293. https://doi.org/10.1007/s10620-009$1007-x$

Petri R, Brattås PL, Sharma Y, et al (2019) LINE-2 transposable elements are a source of functional human microRNAs and target sites. PLoS Genet 15:. https://doi.org/10.1371/journal.pgen.1008036 
Petronacci CMC, García AG, Iruegas EP, et al (2020) Identification of prognosis associated microRNAs in HNSCC subtypes based on TCGA dataset. Med 56:1-10. https://doi.org/10.3390/medicina56100535

Piriyapongsa J, Mariño-Ramírez L, Jordan IK (2007) Origin and evolution of human microRNAs from transposable elements. Genetics 176:1323-1337. https://doi.org/10.1534/genetics.107.072553

Qi P, Wang L, Zhou B, et al (2015) Associations of miRNA polymorphisms and expression levels with breast cancer risk in the Chinese population. Genet Mol Res 14:6289-6296.

https://doi.org/10.4238/2015.June.11.2

Qin S, Jin P, Zhou X, et al (2015) The role of transposable elements in the origin and evolution of microRNAs in human. PLoS One 10:. https://doi.org/10.1371/journal.pone.0131365

Quach H, Barreiro LB, Laval G, et al (2009) Signatures of Purifying and Local Positive Selection in Human miRNAs. Am J Hum Genet 84:316-327. https://doi.org/10.1016/j.ajhg.2009.01.022

R Core Team. R: A language and environment for statistical computing. R Foundation for Statistical Computing. 2020. https://www.r-project.org/.

Rawlings-Goss RA, Campbell MC, Tishkoff SA (2014) Global population-specific variation in miRNA associated with cancer risk and clinical biomarkers. BMC Med Genomics 7:. https://doi.org/10.1186/1755-8794-7-53

Reed ER, Latourelle JC, Bockholt JH, et al (2018) MicroRNAs in CSF as prodromal biomarkers for Huntington disease in the PREDICT-HD study. Neurology 90:E264-E272.

https://doi.org/10.1212/WNL.0000000000004844

Rentzsch P, Witten D, Cooper GM, et al (2019) CADD: Predicting the deleteriousness of variants throughout the human genome. Nucleic Acids Res 47:D886-D894. https://doi.org/10.1093/nar/gky1016 Riffo-Campos ÁL, Riquelme I, Brebi-Mieville P (2016) Tools for sequence-based miRNA target prediction: What to choose? Int. J. Mol. Sci. 17

Sabeti PC, Reich DE, Higgins JM, et al (2002) Detecting recent positive selection in the human genome from haplotype structure. Nature 419:832-837. https://doi.org/10.1038/nature01140

Santpere G, Lopez-Valenzuela M, Petit-Marty N, et al (2016) Differences in molecular evolutionary rates among microRNAs in the human and chimpanzee genomes. BMC Genomics 17:. https://doi.org/10.1186/s12864-016-2863-3

Sarabandi S, Sattarifard H, Kiumarsi M, et al (2021) Association between Genetic Polymorphisms of miR1307, miR-1269, miR-3117 and Breast Cancer Risk in a Sample of South East Iranian Women. Asian Pacific J Cancer Prev 22:201-208. https://doi.org/10.31557/APJCP.2021.22.1.201 
Satoh JI, Kino Y, Niida S (2015) MicroRNA-Seq data analysis pipeline to identify blood biomarkers for alzheimer's disease from public data. Biomark Insights 2015:21-31.

https://doi.org/10.4137/BMI.S25132

Saunders MA, Liang H, Li WH (2007) Human polymorphism at microRNAs and microRNA target sites. Proc Natl Acad Sci U S A 104:3300-3305. https://doi.org/10.1073/pnas.0611347104

Sethupathy P, Collins FS (2008) MicroRNA target site polymorphisms and human disease. Trends Genet 24:489-497. https://doi.org/10.1016/j.tig.2008.07.004

Slattery ML, Mullany LE, Sakoda LC, et al (2018) The MAPK-signaling pathway in colorectal cancer: Dysregulated genes and their association with micrornas. Cancer Inform 17: https://doi.org/10.1177/1176935118766522

Slattery ML, Mullany LE, Sakoda LC, et al (2018) The PI3K/AKT signaling pathway: Associations of miRNAs with dysregulated gene expression in colorectal cancer. Mol Carcinog 57:243-261. https://doi.org/10.1002/mc.22752

Smit, AFA, Hubley, R \& Green, P. RepeatMasker Open-4.0. 2013-2015 <http://www.repeatmasker.org>

Springelkamp H, Iglesias Al, Mishra A, et al (2017) New insights into the genetics of primary open-angle glaucoma based on meta-analyses of intraocular pressure and optic disc characteristics. Hum Mol Genet 26:438-453. https://doi.org/10.1093/hmg/ddw399

Sun X hui, Geng X lin, Zhang J, Zhang C (2015) miRNA-646 suppresses osteosarcoma cell metastasis by downregulating fibroblast growth factor 2 (FGF2). Tumor Biol 36:2127-2134.

https://doi.org/10.1007/s13277-014-2822-z

Sung H, Ferlay J, Siegel RL, et al (2021) Global cancer statistics 2020: GLOBOCAN estimates of incidence and mortality worldwide for 36 cancers in 185 countries. CA Cancer J Clin 71: https://doi.org/10.3322/caac. 21660

Szpiech ZA, Hernandez RD (2014) Selscan: An efficient multithreaded program to perform EHH-based scans for positive selection. Mol Biol Evol 31:2824-2827. https://doi.org/10.1093/molbev/msu211

Torruella-Loran I, Laayouni H, Dobon B, et al (2016) MicroRNA Genetic Variation: From Population Analysis to Functional Implications of Three Allele Variants Associated with Cancer. Hum Mutat 37:1060-1073. https://doi.org/10.1002/humu.23045

Torruella-Loran I, Ramirez Viña MK, Zapata-Contreras D, et al (2019) rs12416605:C>T in MIR938 associates with gastric cancer through affecting the regulation of the CXCL12 chemokine gene. Mol Genet Genomic Med 7:. https://doi.org/10.1002/mgg3.832 
Voight BF, Kudaravalli S, Wen X, Pritchard JK (2006) A map of recent positive selection in the human genome. PLoS Biol 4:0446-0458. https://doi.org/10.1371/journal.pbio.0040072

Wang F, Sun GP, Zou YF, et al (2013) Quantitative assessment of the association between miR-196a2 rs11614913 polymorphism and gastrointestinal cancer risk. Mol Biol Rep 40:109-116.

https://doi.org/10.1007/s11033-012-2039-4

Wang J, Shu H, Guo S (2020) MiR-646 suppresses proliferation and metastasis of non-small cell lung cancer by repressing FGF2 and CCND2. Cancer Med 9:4360-4370. https://doi.org/10.1002/cam4.3062

Wang J, Liu Q, Yuan S, et al (2017) Genetic predisposition to lung cancer: Comprehensive literature integration, meta-analysis, and multiple evidence assessment of candidate-gene association studies. Sci Rep 7:. https://doi.org/10.1038/s41598-017-07737-0

Wang L, Sinnott-Armstrong N, Wagschal A, et al (2020) A MicroRNA Linking Human Positive Selection and Metabolic Disorders. Cell 183:684-701.e14. https://doi.org/10.1016/j.cell.2020.09.017

Wang $\mathrm{M}$, Xiong L, Jiang LJ, et al (2019) miR-4739 mediates pleural fibrosis by targeting bone morphogenetic protein 7. EBioMedicine 41:670-682. https://doi.org/10.1016/j.ebiom.2019.02.057

Wang R, Zhang J, Jiang W, et al (2014) Association between a variant in MicroRNA-646 and the susceptibility to hepatocellular carcinoma in a large-scale population. Sci World J 2014:. https://doi.org/10.1155/2014/312704

Wang X, Chen Q, Wang X, et al (2020) ZEB1 activated-VPS9D1-AS1 promotes the tumorigenesis and progression of prostate cancer by sponging miR-4739 to upregulate MEF2D. Biomed Pharmacother 122:. https://doi.org/10.1016/j.biopha.2019.109557

Wang X, Gao J, Zhou B, et al (2019) Identification of prognostic markers for hepatocellular carcinoma based on miRNA expression profiles. Life Sci 232:. https://doi.org/10.1016/j.Ifs.2019.116596

Wang X, Jiang X, Li J, et al (2020) Serum exosomal miR-1269a serves as a diagnostic marker and plays an oncogenic role in non-small cell lung cancer. Thorac Cancer 11:3436-3447.

https://doi.org/10.1111/1759-7714.13644

Wang YW, Zhang W, Ma R (2018) Bioinformatic identification of chemoresistance-associated microRNAs in breast cancer based on microarray data. Oncol Rep 39:1003-1010.

https://doi.org/10.3892/or.2018.6205

Wang Y, Luo J, Zhang H, Lu J (2016) MicroRNAs in the Same Clusters Evolve to Coordinately Regulate Functionally Related Genes. Mol Biol Evol 33:2232-2247. https://doi.org/10.1093/molbev/msw089

Wheeler BM, Heimberg AM, Moy VN, et al (2009) The deep evolution of metazoan microRNAs. Evol Dev 11:50-68. https://doi.org/10.1111/j.1525-142X.2008.00302.x 
Xiong G, Wang Y, Ding Q, Yang L. (2015) Hsa-mir-1269 genetic variant contributes to hepatocellular carcinoma susceptibility through affecting SOX6. Am J Transl Res ;7(10):2091-2098.

Xu H, Liu X, Zhao J (2014) Down-regulation of mir-3928 promoted osteosarcoma growth. Cell Physiol Biochem 33:1547-1556. https://doi.org/10.1159/000358718

Xu X, Li Z, Liu J, et al (2017) MicroRNA expression profiling in endometriosis-associated infertility and its relationship with endometrial receptivity evaluated by ultrasound. J Xray Sci Technol 25:523-532. https://doi.org/10.3233/XST-17286

Xu YX, Sun J, Xiao WL, et al (2019) MiR-4513 mediates the proliferation and apoptosis of oral squamous cell carcinoma cells via targeting CXCL17. Eur Rev Med Pharmacol Sci 23:3821-3828. https://doi.org/10.26355/eurrev_201905_17809

Yan W, Gao X, Zhang S (2017) Association of miR-196a2 rs11614913 and miR-499 rs3746444 polymorphisms with cancer risk: A meta-analysis. Oncotarget 8:114344-114359. https://doi.org/10.18632/oncotarget.22547

Yang S, Zheng Y, Zhou L, et al (2020) miR-499 rs3746444 and miR-196a-2 rs11614913 Are Associated with the Risk of Glioma, but Not the Prognosis. Mol Ther - Nucleic Acids 22:340-351. https://doi.org/10.1016/j.omtn.2020.08.038

Yang W, Xiao W, Cai Z, et al (2020) miR-1269b drives cisplatin resistance of human non-small cell lung cancer via modulating the PTEN/PI3K/AKT signaling pathway. Onco Targets Ther 13:109-118. https://doi.org/10.2147/OTT.S225010

Zhang P, Tang WM, Zhang H, et al (2017) MiR-646 inhibited cell proliferation and EMT-induced metastasis by targeting FOXK1 in gastric cancer. Br J Cancer 117:525-534. https://doi.org/10.1038/bjc.2017.181

Zhang T, Zhao D, Wang Q, et al (2013) MicroRNA-1322 regulates ECRG2 allele specifically and acts as a potential biomarker in patients with esophageal squamous cell carcinoma. Mol Carcinog 52:581-590. https://doi.org/10.1002/mc.21880

Zhang Z yao, Li Y chen, Geng C ying, et al (2019) Serum exosomal microRNAs as novel biomarkers for multiple myeloma. Hematol Oncol 37:409-417. https://doi.org/10.1002/hon.2639

Zhao H, Xu J, Zhao D, et al (2016) Somatic Mutation of the SNP rs11614913 and Its Association with Increased MIR 196A2 Expression in Breast Cancer. DNA Cell Biol 35:81-87. https://doi.org/10.1089/dna.2014.2785

Zhao M, Dong G, Meng Q, et al (2020) Circ-HOMER1 enhances the inhibition of miR-1322 on CXCL6 to regulate the growth and aggressiveness of hepatocellular carcinoma cells. J Cell Biochem 121:44404449. https://doi.org/10.1002/jcb.29672 
Zhou Y, An H, Wu G (2020) Microrna-6071 suppresses glioblastoma progression through the inhibition of pi3k/akt/ mtor pathway by binding to ulbp2. Onco Targets Ther 13:9429-9441.

https://doi.org/10.2147/OTT.S265791

Zhu K, Wang Y, Liu L, et al (2020) Long non-coding RNA MBNL1-AS1 regulates proliferation, migration, and invasion of cancer stem cells in colon cancer by interacting with MYL9 via sponging microRNA-4123p. Clin Res Hepatol Gastroenterol 44:101-114. https://doi.org/10.1016/j.clinre.2019.05.001

Zhu M, Wang $\mathrm{F}$, Mi H, et al (2020) Long noncoding RNA MEG3 suppresses cell proliferation, migration and invasion, induces apoptosis and paclitaxel-resistance via miR-4513/PBLD axis in breast cancer cells. Cell Cycle 19:3277-3288. https://doi.org/10.1080/15384101.2020.1839700

\section{Tables}

Table 1. Top 5\% miRNA candidates under putative positive selection.

\begin{tabular}{|c|c|c|c|c|c|c|c|c|c|c|}
\hline Chr & Mature ID & Mature SNP & Seed SNP & $\begin{array}{c}\text { Evolutionary } \\
\text { Age }\end{array}$ & $\begin{array}{c}\text { Genomic } \\
\text { Context }\end{array}$ & $\begin{array}{c}\text { Max. } \\
\text { Fst }\end{array}$ & $\begin{array}{l}\text { Max. } \\
\text { iHS }\end{array}$ & $\begin{array}{l}\text { Max. } \\
\text { nSL }\end{array}$ & CADD & Disease association \\
\hline 1 & hsa-miR-4781-3p & - & rs74085143 & Primate & PC;TE & 0.21 & $-/ 1.51$ & $-/ 1.28$ & $-/ 7.85$ & $\mathrm{PD}^{1}, \mathrm{AD}^{2}$ \\
\hline 2 & hsa-miR-6071 & \begin{tabular}{|l|} 
rs56790095 \\
\end{tabular} & - & Primate & $\mathrm{PC} ; \mathrm{TE}$ & 0.21 & $0.67 /-$ & $0.37 /-$ & $5.64 /-$ & $\mathrm{GB}^{3}, \mathrm{CRC}^{4,5}$ \\
\hline 2 & hsa-miR-6811-3p & rs2292879 & - & Primate & $\mathrm{PC} ; \mathrm{TE}$ & 0.26 & $2.73 /-$ & $1.73 /-$ & $2.71 /-$ & - \\
\hline 3 & hsa-miR-6826-5p & rs115693266 & rs6771809 & Primate & $\mathrm{PC}$ & 0.27 & $0.22 / 1.80$ & $0.88 / 2.22$ & $2.92 / 1.01$ & $\mathrm{CRC}^{\gamma}, \mathrm{BC}^{7}$ \\
\hline 4 & hsa-miR-1269a & rs73239138 & - & Primate & $\mathrm{TE}$ & 0.22 & $1.84 /-$ & $2.12 /-$ & $0.70 /-$ & $\begin{array}{c}\mathrm{GC}^{8}, \mathrm{HC}^{9.10}, 16, \mathrm{CC}^{11}, \mathrm{BC}^{12}, \\
\mathrm{LC}^{13,14}, \mathrm{CRC}^{15}\end{array}$ \\
\hline 6 & hsa-miR-10524-5p & - & rs77651740 & Non-classified & TE & \begin{tabular}{|l|}
0.30 \\
\end{tabular} & $-/ 1.69$ & $-/ 1.40$ & $-/ \mathrm{NA}$ & - \\
\hline 8 & hsa-miR-1322 & \begin{tabular}{|l|} 
rs59878596 \\
\end{tabular} & $=$ & Non-classified & PC & \begin{tabular}{|l|}
0.23 \\
\end{tabular} & $1.33 /=$ & $2.25 /=$ & $\mathrm{NA} /=$ & $\mathrm{HC}^{17}, \mathrm{ESC}^{18}$ \\
\hline 8 & hsa-miR-4472 & - & rs28655823 & Primate & Intg & 0.36 & $-/ 2.02$ & $-/ 1.38$ & $-/ 2.87$ & $\mathrm{BC}^{19,20,21}, \mathrm{PC}^{21}, \mathrm{CC}^{21}$ \\
\hline 8 & hsa-miR-8084 & rs 404337 & - & Non-classified & TE & 0.27 & $1.45 /-$ & $1.89 /-$ & $\mathrm{NA} /-$ & $\mathrm{BC}^{22}, \mathrm{OC}^{23}$ \\
\hline 10 & hsa-miR-938 & - & rs 12416605 & Primate & $\mathrm{PC}$ & 0.21 & $-/ 0.93$ & $-/ 1.78$ & $-/ 7.68$ & $\mathrm{GC}^{24,25}$ \\
\hline 11 & hsa-miR-1304-3p & rs2155248 & - & Primate & $\mathrm{PC} ; \mathrm{TE}$ & 0.23 & $1.72 /-$ & $1.13 /-$ & $4.44 /-$ & $\mathrm{GC}^{26}, \mathrm{HC}^{27}, \mathrm{HNC}^{28}, \mathrm{EM}^{29}, \mathrm{LC}^{30}$ \\
\hline 12 & hsa-miR-196a-3p & rs11614913 & - & Eutherians & $\mathrm{PC}$ & 0.24 & $1.74 /-$ & $1.21 /-$ & $18.77 /-$ & $\begin{array}{c}\mathrm{LC}^{31} 138, \mathrm{HC}^{31} 133, \mathrm{HNC}^{31}, \mathrm{GM}^{32}, \\
\mathrm{OC}^{33}, \mathrm{BC}^{33,3537,81}, \mathrm{DM}^{34}, \\
\mathrm{CAD}^{36}, \mathrm{CRC}^{76}, \mathrm{GC}^{77,78,79,30}\end{array}$ \\
\hline 14 & hsa-miR-412-3p & rs61992671 & $=$ & Eutherians & LNC & 0.43 & $1.44 / \cdot$ & $1.33 /=$ & $15.52 /=$ & $\mathrm{OS}^{39}, \mathrm{CC}^{40}$ \\
\hline 14 & hsa-miR-4707-3p & - & rs2273626 & Eutherians & $\mathrm{PC}$ & 0.57 & $-/ 2.33$ & $-/ 1.22$ & $-/ 10.85$ & $\mathrm{POAG}^{41}, \mathrm{ESC}^{42}$ \\
\hline 15 & hsa-miR-4513 & - & rs2168518 & Meta/Prototheria & $\mathrm{PC}$ & 0.59 & $-/ 2.09$ & $-/ 1.40$ & $-/ 5.01$ & $\begin{array}{c}\mathrm{CAD}^{43,46}, \mathrm{LC}^{44,45}, \mathrm{GC}^{47}, \mathrm{BC}^{48}, \\
\mathrm{OSCC}^{49}\end{array}$ \\
\hline 17 & hsa-miR-548h-5p & rs 9913045 & - & Primate & $\mathrm{PC} ; \mathrm{TE}$ & 0.24 & $2.56 /-$ & $3.28 /-$ & $1.31 /-$ & $\mathrm{GM}^{50}$ \\
\hline 17 & hsa-miR-1269b & \begin{tabular}{|r|} 
rs12451747 \\
\end{tabular} & rs7210937 & Primate & PC;TE & 0.33 & $1.67 / 1.11$ & $2.23 / 1.27$ & \begin{tabular}{|l}
$0.31 / 0.39$ \\
\end{tabular} & $\mathrm{OPSCC}^{51}, \mathrm{LC}^{52}$ \\
\hline 17 & hsa-miR-4739 & rs73410309 & - & Primate & LNC;TE & 0.37 & $2.01 /=$ & $3.08 /=$ & $12.73 /=$ & $\begin{array}{c}\mathrm{PF}^{53}, \mathrm{PC}^{54}, \mathrm{DM}^{58.56}, \mathrm{GC}^{57}, \\
\mathrm{AML}^{58}\end{array}$ \\
\hline 18 & hsa-miR-4741 & - & rs7227168 & Eutherians & $\mathrm{PC}$ & 0.27 & $-/ 3.37$ & $-/ 1.74$ & $-/ 13.31$ & $\mathrm{MY}^{59}, \mathrm{HC}^{6}, \mathrm{CRC}^{61}, \mathrm{CC}^{61}$ \\
\hline 19 & hsa-miR-6796-3p & $=$ & rs3745198 & Primate & PC & 0.35 & $-/ 2.39$ & $-/ 1.63$ & $-/ 3.67$ & $\mathrm{UR}^{62}$ \\
\hline 20 & hsa-miR-646 & rs6513497 & - & Primate & LNC;TE & 0.22 & $2.99 /-$ & $3.06 /-$ & $6.33 /-$ & $\begin{array}{c}\mathrm{GC}^{63,68}, \mathrm{HC}^{44}, \mathrm{LAC}^{65}, \mathrm{LC}^{66,71}, \\
\mathrm{BC}^{67}, \mathrm{CRC}^{60}, \mathrm{RC}^{70}, \mathrm{OS}^{72} \\
\end{array}$ \\
\hline 22 & hsa-miR-3928-5p & rs5997893 & - & Non-classified & TE & 0.23 & $1.36 /-$ & $2.01 /-$ & $\mathrm{NA} /-$ & $\mathrm{HD}^{73}, \mathrm{HNC}^{74}, \mathrm{OS}^{75}$ \\
\hline
\end{tabular}

The Max. Fst value represents the maximum mean Fst of the mature sequence among the three comparisons of reference. The selection test values (iHS and nSL) correspond to the population that exhibit the maximum value of the mature SNP (left) and seed SNP (right). The CADD column provides the 
predicted deleteriousness scores of the mature SNP (left) and seed SNP (right). Disease association for most of the candidates are indicated in the disease column and some examples are described in the main text: PD (Parkinson disease), AD (Alzheimer's disease), GB (glioblastoma), CRC (colorectal cancer), ESC (esophageal squamous cell carcinoma), BC (breast cancer), GC (gastric cancer), HC (hepatocellular carcinoma), CC (colon cancer), HNC (head and neck squamous cell carcinoma), EM (endometriosis), LC (lung cancer), POAG (open-angle glaucoma), ESC (esophageal squamous cell carcinoma), GM (glioma), OC (ovarian cancer), DM1 (type 1 diabetes mellitus), CAD (coronary artery disease), OSCC (oral squamous cell carcinoma), OPSCC (oral and pharyngeal squamous carcinoma), PF (pleural fibrosis), PC (prostate cancer), AML (acute myeloid leukemia), MY (myeloma), UR (urolithiasis), LAC (laryngeal carcinoma), RC (renal carcinoma), OS (osteosarcoma), HD (Huntington disease). (1) Beecham et al. 2015, (2) Satoh et al. 2015, (3) Zhou et al. 2020, (4,5) Slattery et al. 2018,(6) Kijima et al. 2017, (7) Danková et al. 2020, (8) Li et al. 2017, (9) Min et al. 2017, (10) Xiong et al. 2015, (11) Mao et al. 2017, (12) Sarabandi et al. 2021, (13) Jin et al. 2018, (14) Wang et al. 2020, (15) Bu et al. 2015, (16) Wang et al. 2019, (17) Zhao et al. 2020, (18) Zhang et al. 2013, (19) Li et al. 2020, (20) Wang et al. 2018, (21) Kim et al. 2012, (22) Gao et al. 2018, (23) Chong et al. 2015, (24) Torruella-Loran et al. 2019, (25) Arisawa et al. 2012, (26) Kurata and Lin 2018, (27) Oura et al. 2019, (28) Petronacci et al. 2020, (29) Xu et al. 2017, (30) Othman et al. 2013, (31) Liu et al. 2018, (32) Yang et al. 2020, (33) Choupani et al. 2019, (34) Ibrahim et al. 2019, (35) Ahmad and Shah 2020, (36) Fragoso et al. 2019, (37) Zhao et al. 2016, (38) Wang et al. 2017, (39) Martin-Guerrero et al. 2018, (40) Zhu et al. 2020, (41) Ghanbari, et al. 2017, (42) Bi et al. 2020, (43) Mir et al. 2019, (44) Ghanbari M et al. 2014, (45) Ghanbari M et al. 2017, (46) Li et al. 2015, (47) Ding et al. 2019, (48) Li et al. 2019, (49 Xu et al. 2019, (50) Ji et al. 2020, (51) Chen et al. 2016, (52) Yang et al. 2020, (53) Wang et al. 2019, (54) Wang et al. 2020, (55) Delić et al. 2016, (56) Li et al. 2018, (57) Dong et al. 2015, (58) Cattaneo et al. 2015, (59) Zhang et al. 2019, (60) Liu et al. 2019, (61) Cojocneanu et al. 2020, (62) Liang et al. 2019, (63) Cai et al. 2016, (64) Wang et al. 2014, (65) Yuan et al. 2020, (66) Wang et al. 2020, (67) Darvishi et al. 2020, (68) Zhang et al. 2017, (69) Dai et al. 2017, (70) Li et al. 2014, (71) Pan et al. 2016, (72) Sun et al. 2015, (73) Reed et al. 2018, (74) Fadhil et al. 2020, (75) Xu et al. 2014, (76) Yan et al. 2017, (77) Ni et al. 2015, (78) Yan et al. 2017, (79) Peng et al., 2010, (80) Wang et al 2013, (81) Qi et al. 2015.

Table 2. TargetScanHuman predicted target genes for the seed-variant miRNA candidates 


\begin{tabular}{|c|c|c|c|c|c|c|c|}
\hline Mature ID & SNP & AA & DA & $\begin{array}{l}\text { Targets } \\
\text { (AA) }\end{array}$ & $\begin{array}{l}\text { Targets } \\
\text { (DA) }\end{array}$ & $\begin{array}{l}\text { Overlapping } \\
\text { targets }\end{array}$ & $\begin{array}{l}\text { Cosine } \\
\text { similarity }\end{array}$ \\
\hline hsa-miR-938 & rs12416605 & $\mathrm{C}$ & $\mathrm{T}$ & 2678 & 2594 & 573 & 0.22 \\
\hline hsa-miR-4472 & rs28655823 & G & C & 3257 & 835 & 322 & 0.19 \\
\hline hsa-miR-4513 & rs2168518 & G & A & 2532 & 2693 & 2118 & 0.81 \\
\hline $\begin{array}{l}\text { hsa-miR- } \\
1269 \mathrm{~b}\end{array}$ & rs7210937 & G & C & 2437 & 3167 & 626 & 0.23 \\
\hline $\begin{array}{l}\text { hsa-miR-4707- } \\
3 p\end{array}$ & rs2273626 & $\mathrm{C}$ & A & 1167 & 2592 & 356 & 0.20 \\
\hline hsa-miR-4741 & rs7227168 & $\mathrm{C}$ & $\mathrm{T}$ & 3665 & 2231 & 676 & 0.23 \\
\hline $\begin{array}{l}\text { hsa-miR-4781- } \\
3 p\end{array}$ & rs74085143 & $A$ & G & 2339 & 2724 & 558 & 0.22 \\
\hline $\begin{array}{l}\text { hsa-miR-6796- } \\
3 p\end{array}$ & rs3745198 & C & G & 2331 & 2855 & 484 & 0.19 \\
\hline $\begin{array}{l}\text { hsa-miR-6826- } \\
5 p\end{array}$ & rs6771809 & C & $\mathrm{T}$ & 3191 & 2032 & 517 & 0.20 \\
\hline $\begin{array}{l}\text { hsa-miR- } \\
10524-5 p\end{array}$ & rs77651740 & G & $\mathrm{T}$ & 2853 & 3332 & 2234 & 0.72 \\
\hline
\end{tabular}

Two sets of target genes were predicted for each candidate holding both ancestral (AA) and derived alleles (DA). The overlap between these two lists of target genes is provided and the similarity is estimated with the cosine similarity.

\section{Figures}


a

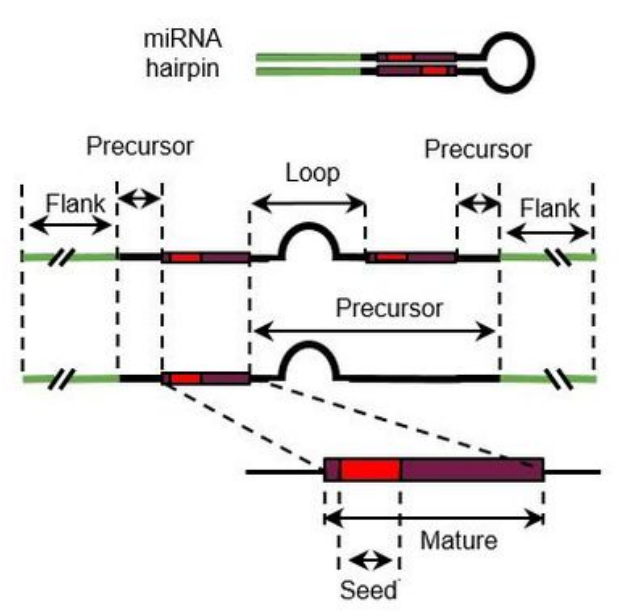

d

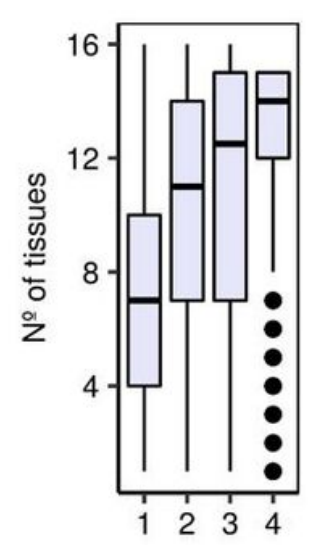

e

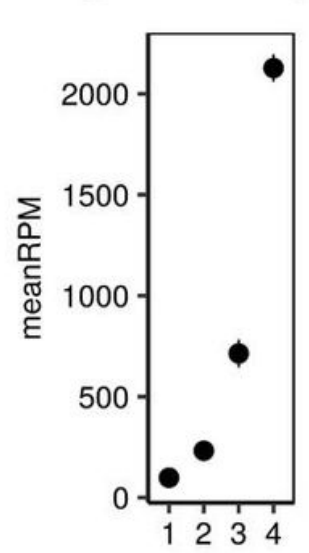

b
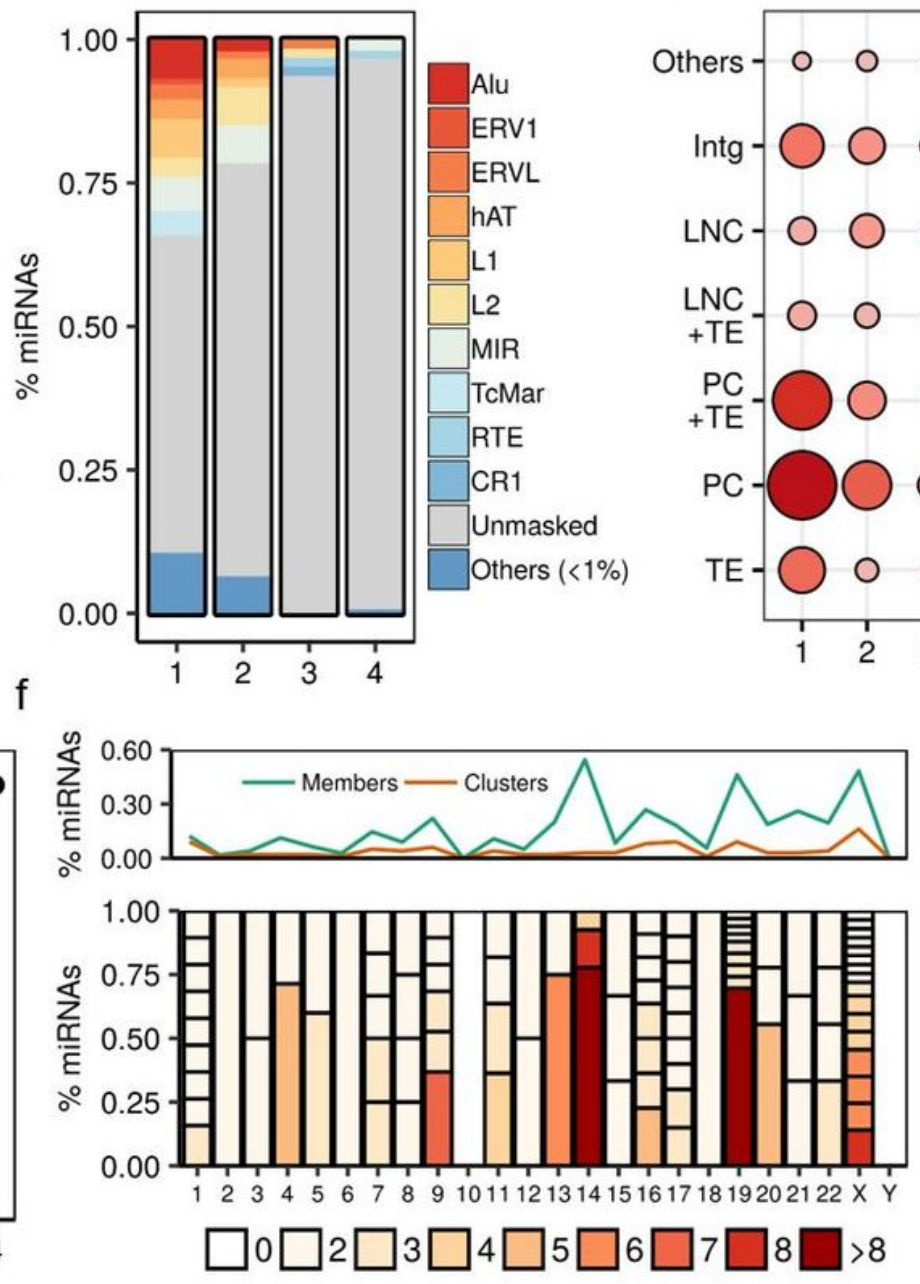

C
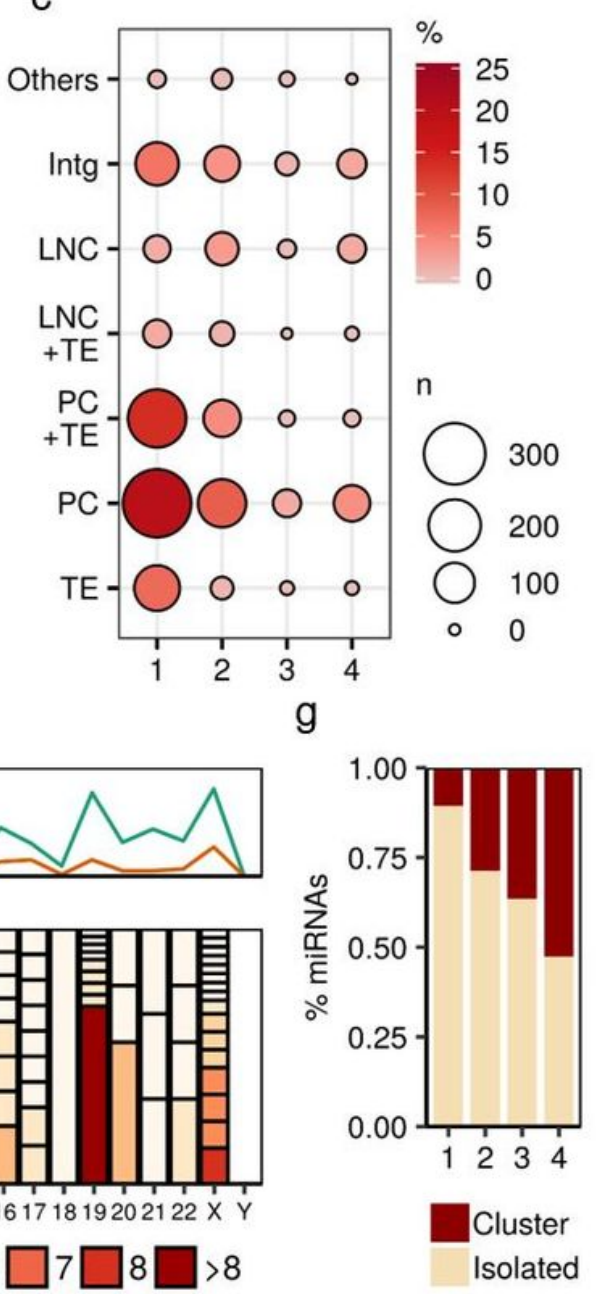

Figure 1

Description of human miRNAs in terms of genomic context, evolutionary age groups, expression levels and clustering. (a) Description of the miRNA hairpin regions identified and analysed in the study. Not all the primary sequences present two mature sequences annotated by miRbase. When the two mature sequences are not given (incomplete annotation), the precursor region is extended from the first mature to the other flanking (Flank) region. (b) TE-derived miRNA frequencies across conservation groups (Primates, 1; Eutherians, 2; Metatheria and Prototheria, 3; Conserved beyond mammals, 4). (c) Integrated hosting of miRNAs showing the combination of the different hosting elements that overlap with miRNA sequences. The "Others" group is made with the minor categories (PC+LNC and PC+LNC+TE) that represent less than $1 \%$ of the total dataset (Supplementary Table S2). (d) Number of tissues where the miRNA is expressed across evolutionary ages (e) Mean expression level on reads per million (RPM) of miRNAs across evolutionary ages (f) Whole genome clustering patterns of miRNAs. The upper plot represents the frequency of miRNAs that belong to a certain cluster in each chromosome (Members) and the frequency of clusters in the whole genome (Clusters). The lower plot represents the miRNA clusters per chromosome, according to the number of members and their frequency among the clustered miRNAs. 
(g) Fraction of clustered and isolated miRNAs across evolutionary ages. Intg (Intergenic), LCN (long noncoding RNA), TE (transposable element), PC (protein-coding).

a

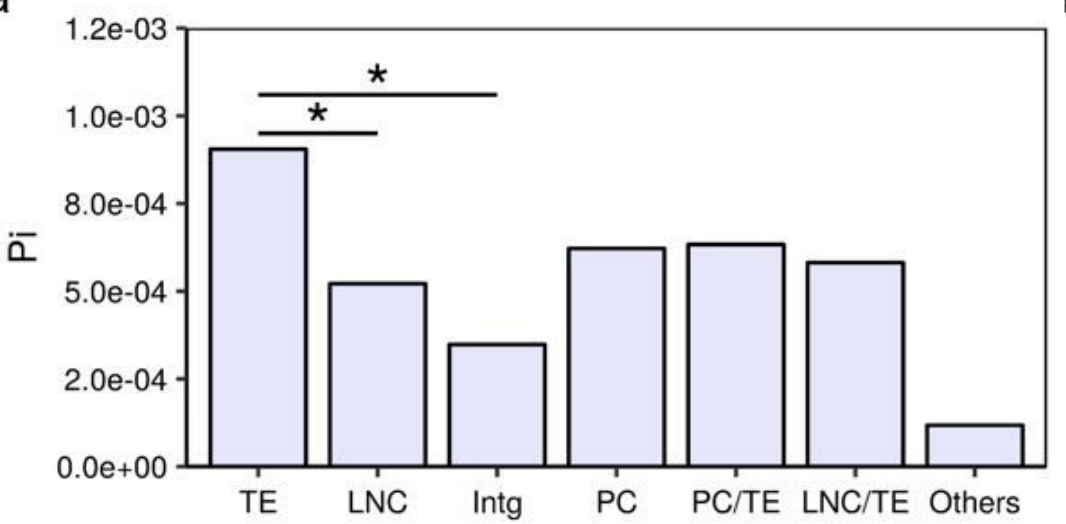

b

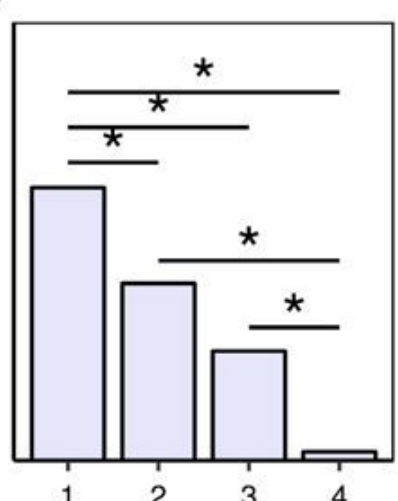

f
C

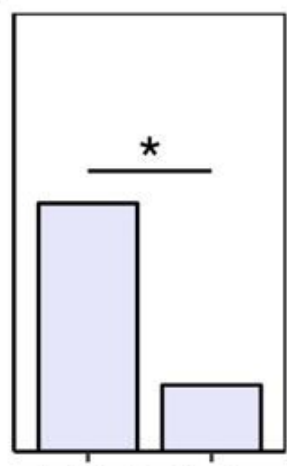

Isolated Cluster
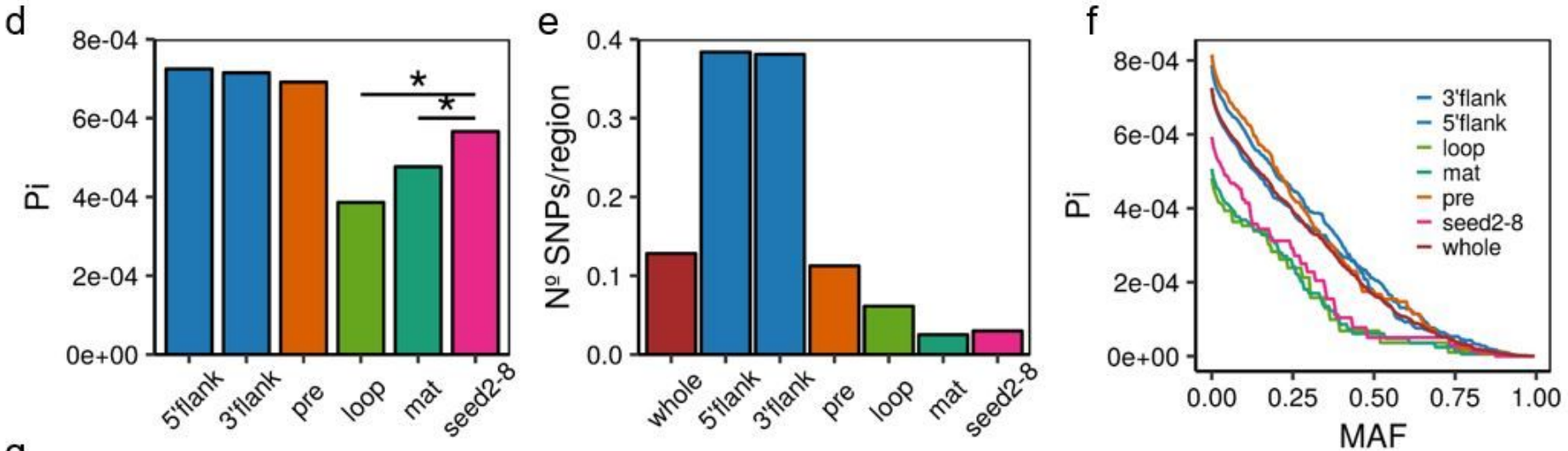

g

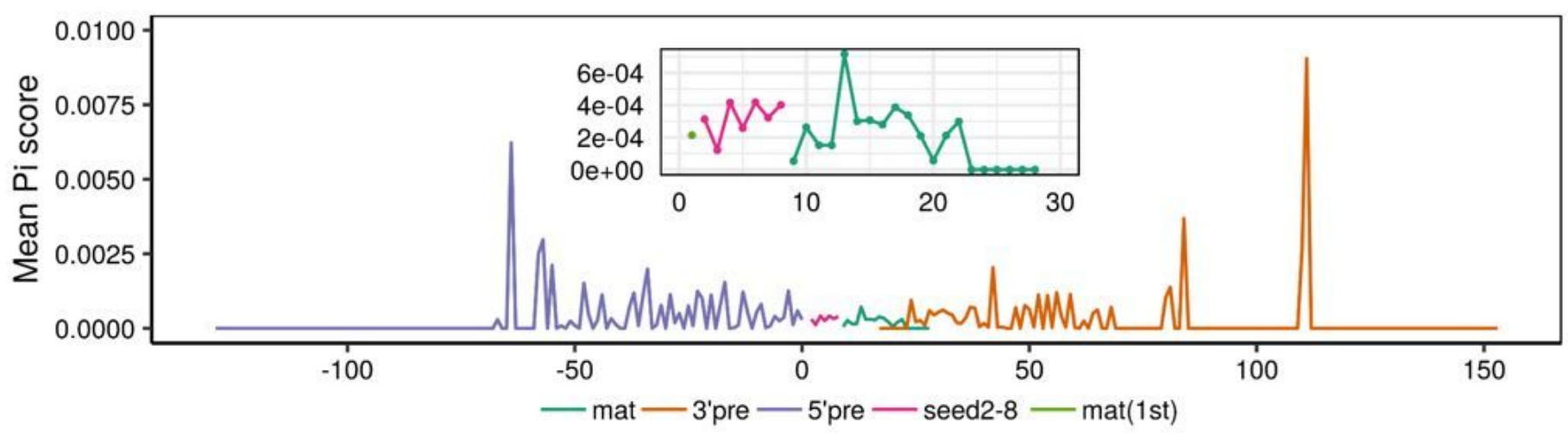

Figure 2

Mean nucleotide diversity differences between miRNAs in different annotation categories and functional regions. (a) Differences between the genomic contexts where the human miRNAs are found. Wilcoxon pairwise comparisons (Bonferroni corrected) show that TEs present a significantly higher diversity than other environments (TE vs $L N C, p=0.022 ; T E$ vs Intg, $p=0.022$. (b) Differences across miRNA conservation groups. Primate-specific miRNAs (group 1 ) show a significantly higher diversity in comparison with the others ( 1 vs $2, p=5.7 e-04 ; 1$ vs $3, p=0.0178$; 1 vs $4, p=3.93 e-10$; Wilcoxon pairwise comparisons, Bonferroni corrected). Significant differences are also seen for the miRNAs conserved beyond mammals (group 4) (4 vs 3, $p=0.0178 ; 4$ vs 2, $p=2.6 e-05$; Wilcoxon pairwise comparisons, 
Bonferroni corrected). (c) Differences between miRNAs found isolated and organised in clusters. Isolated miRNAs are associated with a significantly higher diversity than the members of clusters (Wilcoxon pairwise comparisons, $p=3.7 e-10)$. (d) Diversity comparison between the different functional regions identified in the miRNA hairpins. The seed region (2-8 nucleotides) presents a significantly higher diversity than other conserved regions (seed vs loop, $p=1.1 \mathrm{e}-03$ and seed vs mat, $p=5.6 \mathrm{e}-03$; Wilcoxon pairwise comparisons, Bonferroni corrected). (e) SNP density per functional region calculated in the whole miRNA dataset. (f) Mean nucleotide diversity of the miRNA functional regions across the SNP Minimum Allele Frequency (MAF) range. (g) Mean nucleotide diversity calculated in each relative position of the precursor miRNA. The zoomed region corresponds to the diversity per position found in the mature sequence. TE (transposable element), LCN (long non-coding RNA), Intg (Intergenic), PC (protein-coding), flank (flanking region), pre (precursor), mat (mature).

a

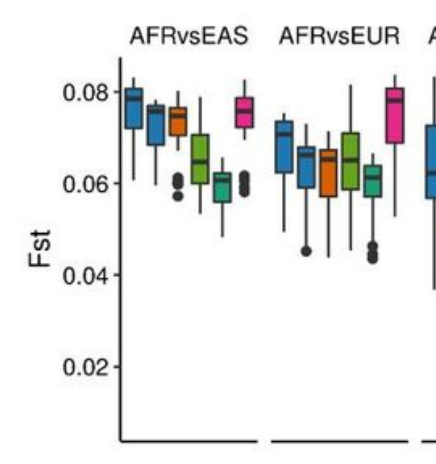

C

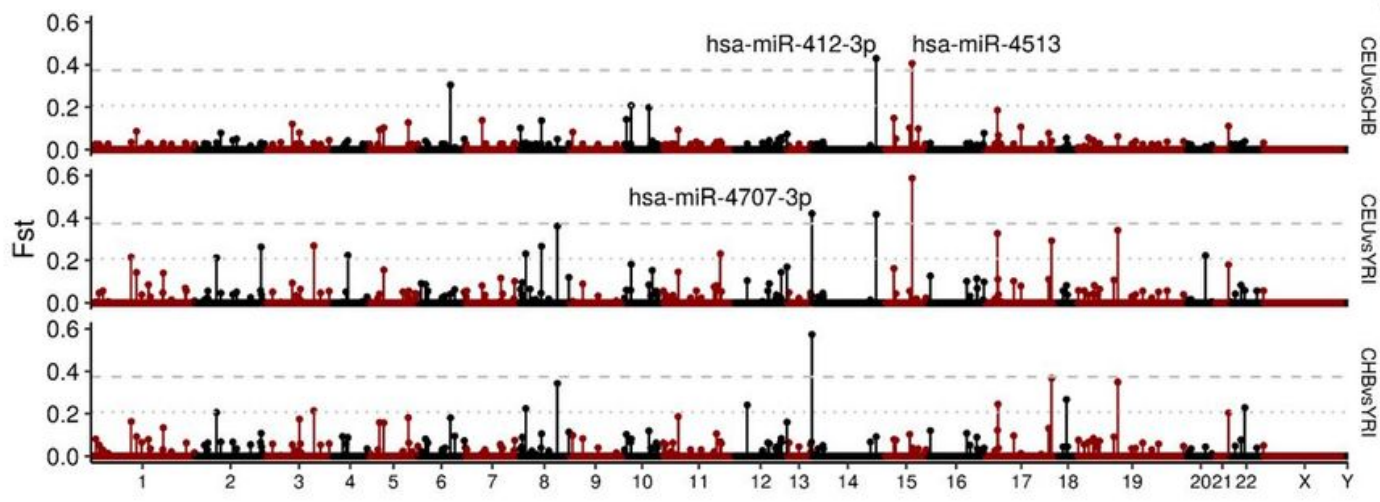

b

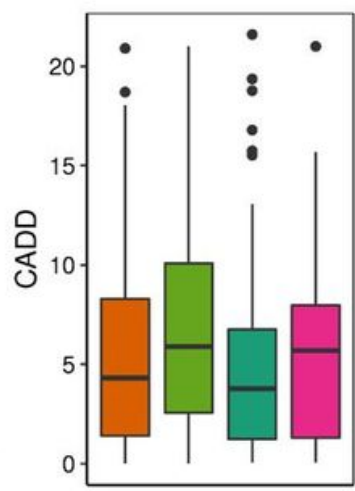

d

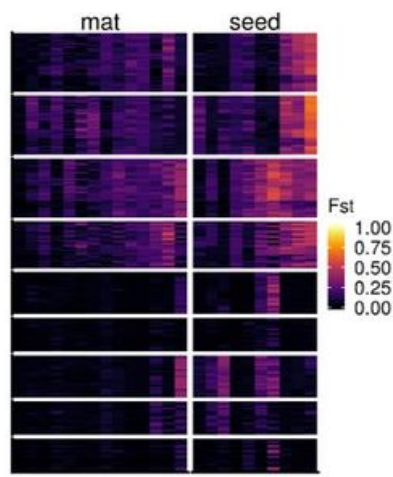

\section{Figure 3}

Analysis of Fst values across miRNA regions and candidates. (a) Mean Fst values per miRNA region across population comparison groups. The Fst values were calculated in all the variant regions. (b) Combined Annotation Dependent Depletion (CADD) scores distributions, as a measure of the predicted level of deleteriousness of the variants, across miRNA regions (c) Manhattan plot showing the mean Fst values per miRNA mature sequence in the three population comparisons of reference. Two Fst thresholds were used to extract the potential miRNA candidates under positive selection (1\% and 5\%). (d) Heatmap showing the per-SNP Fst values of the variants found in the mature outside seed (14) and seed (10) 
regions of the top 5\% miRNA candidates, where the columns correspond to SNPs and rows to all 243 possible population comparisons.

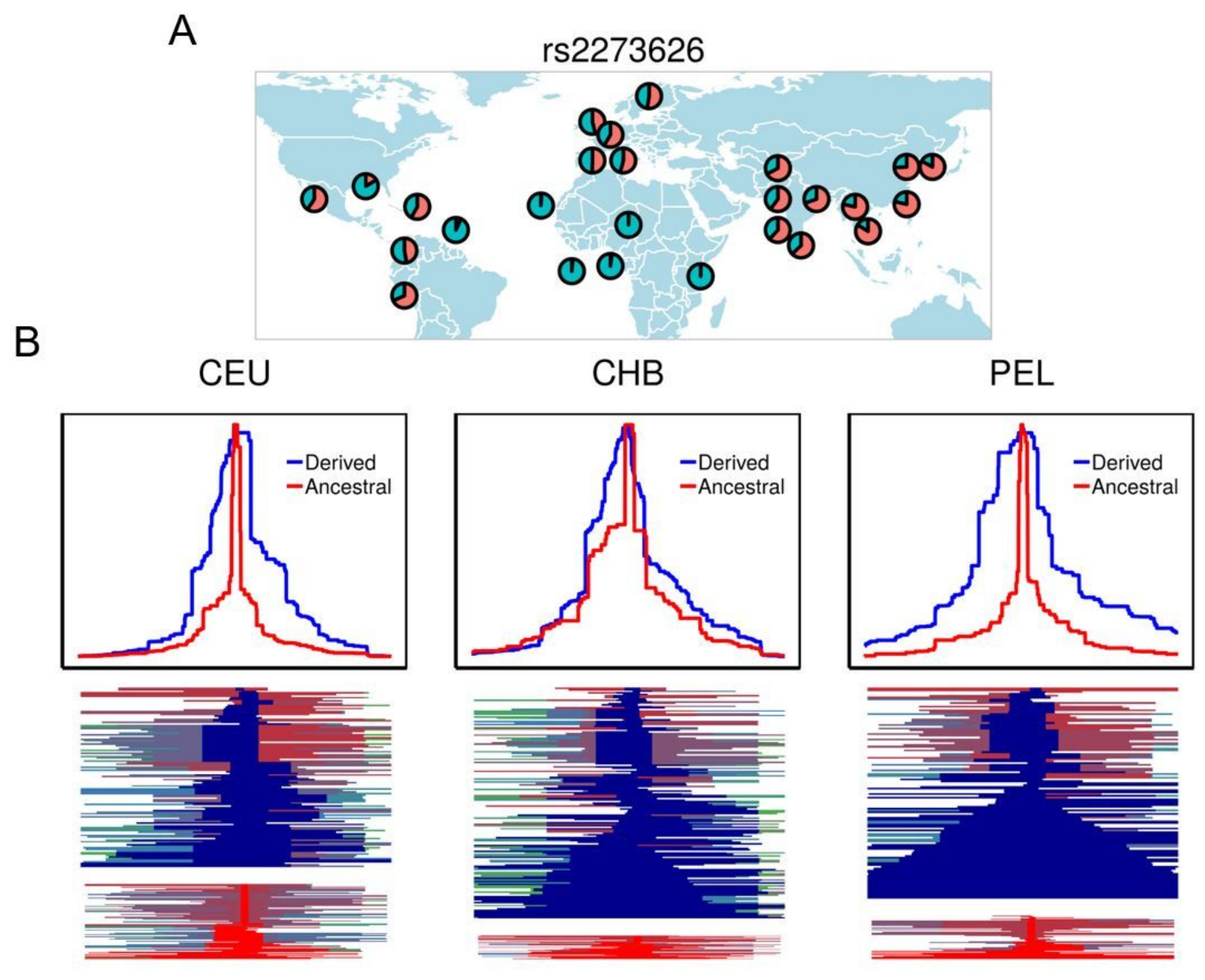

Figure 4

Analysis of signatures of positive selection in the candidate SNP rs2273626 in miR-4707-3p. (a) World wide Minimum Allele Frequency (MAF) distribution of rs2273626. (b) Extended haplotype homozygosity $(E H H)$ decay in both ancestral and derived alleles of rs2273626 (upper plot) and haplotype patterns around the ancestral and derived alleles (bottom plot) in Utah Europeans (CEU), Han Chinese (CHB) and Peruvian (PEL) populations. Note: The designations employed and the presentation of the material on this map do not imply the expression of any opinion whatsoever on the part of Research Square concerning the legal status of any country, territory, city or area or of its authorities, or concerning the delimitation of its frontiers or boundaries. This map has been provided by the authors.

\section{Supplementary Files}


This is a list of supplementary files associated with this preprint. Click to download.

- SupplementaryFile1.HGVillegasMiron.pptx

- SupplementaryFile2HGVillegasMiron.xIsx 